UC-20f

Issued: September 1986

LA- - 10818-MS

DE87 002245

\title{
FRC Formation Experiments with Tearing Reconnection on the FRX-C/LSM Device
}

\author{
D. J. Rej \\ W. N. Hugrass \\ G. A. Barnes \\ R. E. Siemon
}

\section{DISCLAIMER}

This report was prepared as an account of work sponsored by an agency of the United States Government. Neither the United States Government nor any agency thereof, nor any of their employees, makes any warranty, express or implied, or assumes any legal liability or responsibility for the accuracy, completeness, or usefulness of any information, apparatus, product, or process disclosed, or represents that its use would not infringe privately owned rights. Reference herein to any specific commercial product, process, or service by trade name, trademark, manufacturer, or otherwise does not necessarily constitute or imply its endorsement, recommendation, or favoring by the United States Government or any agency thereof. The views and opinions of authors expressed herein do not necessarily state or reflect those of the United States Government or any agency thereof. 


\title{
FRC FORMATION EXPERIMENTS WITH TEARING RECONNECTION ON THE FRX-C/LSM DEVICE
}

\author{
D. J. Rej, W. N. Hugrass, G. A. Barnes and R. E. Siemon
}

\begin{abstract}
Field-reversed configuration (FRC) formation studies performed on the FRX-C/LSM device during February and March 1986 are documented. Experiments were restricted to the tearing-reconnection mode of formation at 10 mtorr deuterium fill pressure. Magnetic tearing was driven with 0.3$\mathrm{m}$-long, 0.66 - $\mathrm{m}$-id passive end mirrors mounted on a $\theta$-pinch coil with a 1.4-m-long, 0.76-m-id central section. Typical plasma densities and temperatures were $\bar{n} \simeq 2 \times 10^{15} \mathrm{~cm}^{-3}$ and $200 \leq T_{e}+T_{i} \leq 450 \mathrm{eV}$, respectively. Two parameters were varied: the magnitude of the reversed-bias B-field, $0.5 \leq B_{b} \leq 1.4 \mathrm{kG}$, and the time interval $\Delta \tau_{P I}$ (set at either 5,12 , or $30 \mu \mathrm{s}$ ) between preionization and the initiation of the main discharge. As $\mathrm{B}_{b}$ was increased the axial shock and radial swelling after reconnection became stronger with transient plasma elongations decreasing from 3 to 1 . The inferred trapped poloidal flux $\Phi_{p}$ increased with $B_{b}$, while at a given $\mathrm{B}_{b}$, the maximum $\Phi_{p}$ was obtained at the shortest $\Delta \tau_{P I}$. The normalized separatrix radius $x_{s}$ at the start of the equilibrium phase varied from 0.35 to 0.65 and $\Phi_{p}$ ranged between 2 and $20 \mathrm{mWb}$. This upper value of $\Phi_{p}$ is about 4-times larger than that achieved in FRX-C with a 0.5 -m-id coil; however, the longest flux confinement times, $\tau_{\Phi} \leq 100 \mu$ s, were obtained only at small $\Phi_{p}$.
\end{abstract}




\section{Introduction and Summary}

The formation of field-reversed configuration (FRC) compact toroid plasmas has been the subject of many recent experimental ${ }^{1-4}$ and theoretical $^{5-9}$ programs. FRC formation with the tearing-reconnection mode of operation has been studied recently in the FRX-C device at Los Alamos, ${ }^{10,11}$ and several important features have been found. For example, as the magnitude of the reversed-bias held $B_{b}$ was increased, more severe axial contractions occurred after magnetic reconnection. Poor confinement of the inferred trapped poloidal fux $\Phi_{p}$ was observed whenever the minimum plasma elongation $\varepsilon_{\min }$ during formation fell below a threshold of about three. ${ }^{10}$

The purpose of this Report is to document similar experimental FRC formation studies performed on the larger FRX-C/LSM device during February and March 1986. The organization of this paper is as follows: In Sec. II the FRX-C/LSM hardware and the experimental conditions are described. Experiments have been restricted to a 10-mtorr, deuterium initial fill pressure while two parameters, $B_{\mathrm{b}}$ and $\Delta \tau_{P I}$, the time interval between preionization (PI) and initiation of the main capacitor bank, were varied. The PI conditions are reviewed in Sec. III. The main bank is discharged with PI integral density profiles that can be peaked at the ends, relatively uniform, or peaked near the center of the $\theta$-pinch coil. FRC formation is reported in Sec. IV. As $B_{b}$ is increased from 0.55 to $1.4 \mathrm{kG}$ stronger axial contractions are observed, while $\Phi_{p}$ increases from 2 to 20 $\mathrm{mWb} . \varepsilon_{\min }$ is usually less than 3 for all conditions.

Observations made during the FRC equilibrium pliase are reported in Sec. V. Larger-radii (or $\Phi_{p}$ ) plasmas appear more hollow. Almost every FRC equilibrium extends axially such that interactions with the endmirrors are probably non-negligible.

A preliminary report on the flux confinement is found in Sec. VI. Best confinement $\left(\tau_{\Phi}>100 \mu \mathrm{sec}\right)$ is found at low $B_{b}$ and with uniform axial profiles of the PI density. Confinement is clearly degraded at the conditions which result in the largest $\Phi_{p}$; however, it is unclear whether this degradation is either due to the formation asymmetries associated with tearing reconnection or due to instabilities which appear at large $\Phi_{p}$. 


\section{Experimental Conditions}

Experiments have been performed on the FRX-C/LSM device, a fieldreversed $\theta$-pinch experiment located at Los Alamos. The experimental mission and the design details of FRX-C/LSM are reviewed elsewhere. ${ }^{12,13}$ The data reported in this paper have been obtained with a $\theta$-pinch coil consisting of a $1.4-\mathrm{m}$-long, $0.76-\mathrm{m}$-diam straight section with $0.3-\mathrm{m}$-long, 0.66 -m-diam passive end mirrors. Typical vacuum magnetic flux surfaces and a field profile are illustrated in Fig. 1. Experiments have been restricted to the following conditions: (1) an initial deuterium fill pressure of 10 mtorr; (2) a $\theta$-pinch preionization bank charge voltage of $45 \mathrm{kV}$ (corresponding to a vacuum peak-to-peak $B$-field modulation of $1.6 \mathrm{kG}$ at the axial midplane; (3) use of a $10-\mathrm{MHz}$ if pre-preionization. The if system consists of a 1.2ms-long, 3-kW pulse transmitted from antennas mounted at each end of the $\theta$-pinch coil on the outside of the 0.6 -m-diam quartz vacuum chamber. Two parameters have been varied in these experiments: (1) the magnitude $B_{b}$ of the reversed-bias magnetic field; and (2) the time interval $\Delta \tau_{P I}$ between the discharging of the $\theta$-pinch preionization and the main capacitor banks. Bias scans with $0.5<B_{b}<1.4 \mathrm{kG}$ have been performed with $\Delta \tau_{P I}$ fixed at either 5,12 , or $30 \mu \mathrm{s}$. The corresponding Green-Newton bias field ${ }^{14}$ at 10 mitorr is $2.1 \mathrm{kG}$.

The two principal plasma diagnostics are an excluded flux array consisting of an external flux loop and twelve magnetic probes and a sixchord HeNe-laser interferometer system. The interferometers view the plasma side-on and they are configured either axially at positions $z=0.0$, $\pm 0.4,+0.6, \pm 0.8 \mathrm{~m}$ (where $z=0$ is the axial midplane) or radially at $z=$ 0 and impact parameters $\mathrm{b}=0,0.06,0.09,0.12,0.15,0.17 \mathrm{~m}$.

Typical FRC plasma parameters obtained are: $n_{e} \simeq 2 \times 10^{15} \mathrm{~cm}^{-3}, \mathrm{~T}_{e}$ $+\mathrm{T}_{i}=200 \rightarrow 450 \mathrm{eV}, B_{w}=4 \rightarrow 6 \mathrm{kG}, x_{s}=0.4 \rightarrow 0.6$.

\section{Preionization}

The preionization (PI) of the 10 -mtorr- $\mathrm{D}_{2}$ fill has been characterized with the excluded flux and interferometer arrays. The magnetic data are incomplete since the internal B-field is not measured. Internal measure- 
ments are needed to infer the movement of a plasma sheath during a PI discharge ${ }^{15}$ however, the external measurements discussed below provide useful information. Typical signals from the center interferometer chord $(b=0, z=0)$ are illustrated with the corresponding excluded flux data in Figs. 2 and 3 for PI discharges at low $\left(B_{b} \simeq 0.7 \mathrm{kG}\right)$ and moderate $\left(B_{b} \simeq 1.15 \mathrm{kG}\right)$ bias strengths, respectively. In these figures the time $t=0$ corresponds to the discharging of the $\theta$-pinch PI capacitor bank.

The time of the initial gas breakdown depends on $B_{b}$. From the data in Figs. 2 and 3 this breakdown is first detected by the initial appearance of excluded flux $\Delta \Phi$. The integral density at this time is usually too small to observe with the interferometers. At low bias (Fig. 4) breakdown occurs at time $t \simeq 1.8 \mu \mathrm{s}$, at about the time of the second zero-crossing when $B<B_{b}$ and $E_{\theta}$ is at about half-maximum. At the largest bias (Fig. 4), breakdown occurs at larger fields, $B \geq B_{b}$. Evidently, there appears to exist a transition field $B_{b} \approx 1.1 \mathrm{kG}$, (somewhat above the zero-crossing field of $B_{b}=0.8 \mathrm{kG}$ ) above which initial breakdown occurs near the applied bias level. the magnitude and spatial dependence of the integral density early in time vary with the bias strength. At lower bias, $B_{b}<1.1 \mathrm{kG}$, a measurable plasma density first appears at time $t \simeq 4 \mu$ s near the ends of the $\theta$-pinch coil. These end plasmas are radially compressed by time $t \simeq 5 \mu \mathrm{s}$. The midplane plasma is compressed radially a couple of microseconds later. Sample $\int n d l$ profiles are illustrated in Figs. 5 and 6 for the $B_{b} \simeq 0.7 \mathrm{kG}$ case. The plasma at the axial midplane does not undergo a second radial compression even though the B-field oscillates a complete cycle between $t=10$ and $15 \mu \mathrm{s}$. Density waves do, however, propagate axially toward the midplane between $t=15$ and $20 \mu \mathrm{s}$ which results in a second, more modest radial compression or plasma accumulation at the center. The axial $\int n d l$ profile remains peaked until after $t=30 \mu \mathrm{s}$. As $B_{b}$ is increased from 0.6 to $1.0 \mathrm{kG}$ the initial axial profile becomes less peaked while the initial radial compression at the midplane and subsequent axial density waves become less pronounced.

At higher bias, $B_{b} \geq 1.1 \mathrm{kG}$ the integral density profile at the midplane is relatively uniform for all times (Fig. 7). The axial profile (Fig. 8) is also nore uniform early in time but becomes peaked after weak density waves reach the center at $t=22 \mu \mathrm{s}$. At the highest bias, $B_{b} \geq 1.25$, the profiles 
remain relatively uniform but the degree of ionization is further reduced and varies from discharge-to-discharge.

The Milroy-Brackbill 2-D MHD ${ }^{16}$ code has been used to model the preionization plasma. The initial conditions for the sinulations are very idealized. The plasma is assumed to be fully ionized and uniform over the tube cross section. The initial density is assumed to decrease linearly with the axial distance outside the coil. The calculation starts $2.2 \mu$ s after the preionization bank is fired; this is approximately the time when the observed excluded flux signal becomes significant (see Fig. 2). The flux in the coil is approximated by:

$$
\Phi(t)=\pi r^{2} B_{b}+\pi r^{2} B_{P I} \sin 2 \pi f\left(t-t_{o}\right) e^{-\left(t-t_{o}\right) / \tau}
$$

where $r$ is the radius of the coil, $B_{b}$ is the bias field $(0.8 \mathrm{kG}$ in the central section), $\mathrm{B}_{P_{I}}$ is the initial amplitude of the preionization magnetic field $(1.0 \mathrm{kG}), \mathrm{f}$ is the frequency of the preionization field $(170 \mathrm{KHz}), \mathrm{t}_{o}$ is the breakdown time $(2.2 \mu \mathrm{sec})$ and $\tau$ is the decay time constant of the preionization waveform $(6.0 \mu \mathrm{sec})$. The purpose of these calculations is to show the qualitative features of the preionization plasma. No attempt has been made to vary the simulation parameters so that the calculation results are in quantitative agreement with the experimental observations. Figure 9a shows the velocity vectors and the flux contours in the upper left quadrant for $\mathrm{t}=3.2,4.2,6.2,8.2,10.2,12.2$ and $14.2 \mu \mathrm{s}$. It is seen that the oscillating field excite a compressional wave which propagates approximately in the radial direction. Both the amplitude and propagation velocity of this wave are larger under the passive mirror region than in the central section. The wave motion is a mixture of the natural oscillation of the plasma column in response to the early large amplitude perturbation and a fast decaying forced oscillation. The complexity of this motion leads to continuous variation of the flow patterns. Note also the gradual increase of the directed axial flow outside the coil. Figure $9 \mathrm{~b}$ shows the variation of the line density and $\int \mathrm{ndl}$ for $\mathrm{t}=3.2,4.2,6.2,8.2,10.2,12.2$, and $14.2 \mu \mathrm{sec}$. Note that $\int \mathrm{ndl}$ as measured by a side-on, two-pass interferometer equals $4 \int_{0}^{R} \mathrm{n}(\mathrm{r}) \mathrm{dr}$. It is seen that the line density under the mirror section decreases steadily as the plasma is evacuated from this region. $\int \mathrm{ndl}$ exhibits larger variations as the plasma experiences radial oscillations which have 
different phases at different axial locations because $\int \mathrm{ndl}$ depends on the radial density distribution.

\section{FRC Formation}

The formation of the FRC equilibrium is accomplished using almost the same sequence as that used in earlier experiments on FRX-C ${ }^{10}:(1)$ application of reversed bias field; (2) rf pre-preionization; (3) ringing- $\theta$-pinch preionization; (4) discharge of the main capacitor bank which results in: (a) field-reversal and plasma lift-off from the wall; (b) radial compression; (c) magnetic reconnection at the ends and axial contraction. This sequence is revealed by the magnetic and interferometric data in Figs. 10-13 for typical discharges with $B_{6} \simeq 0.7 \mathrm{kG}$ and $\Delta \tau_{P I} \simeq 12 \mu \mathrm{s}$. (The best flux confinement to date has been obtained at these conditions - see Sec. VI.) For these data, the main bank is discharged at $t=0$. The "lift-off" B-field is estimated with the "zero-crossing" field, $B_{z c} \simeq \Phi(B=0) / \pi r_{t}^{2}$, where $r_{t}$ is the vacuun chamber wall radius and $\Phi(B=0)$ is the flux measured at the instant the external B-field crosses zero. As illustrated in Fig. 14, $B_{z c}$ increases with $B_{b}$ and depends on $\Delta \tau_{P I}$. The zero-crossing field increases nonlinearly with the bias strength. At a given $B_{b}$ there is little change in $B_{z c}$ at the two early PI timings, $\Delta \tau_{P I}=12$ or $30 \mu \mathrm{s}$; however, the zero-crossing field is clearly larger at delayed PI, $\Delta \tau_{P I} \simeq 5 \mu \mathrm{s}$. As $B_{b}$ is increased from 0.6 to $1.3 \mathrm{kG}$, the ratio of trapped to applied bias flux at reversal $B_{z c} / B_{b}$ varies from $15 \%$ to $50 \%$ at early PI and $65 \%$ to $85 \%$ at delayed PI. It is unclear at this time on the cause of the $B_{z c}$ increase at short $\Delta \tau_{P I}$. These observations might be attributed to smaller flux losses because of the short time interval. On the other hand, the $B_{z c}$ increase might simply be due to the $\theta$-pincl PI B-field augnenting $B_{b}$. For $\Delta \tau_{P I} \simeq 5 \mu \mathrm{s}$, the main bank is discharged during the second-half cycle of the PI waveform at a time when the external $\mathrm{B}$-field is clearly greater than $B_{b}$. Internal B-field measurements are clearly warranted in future studies in order to resolve these observations.

The Fig. 11 and 12 data indicate that the plasma diamagnetism and integral density are peaked at the ends during the radial compression $(4 \leq t \leq$ $7 \mu \mathrm{s})$. The integral density profile contracts axially reaching its minimum length, $l_{\min } \simeq 0.8 \mathrm{~m}$, at time $t \simeq 10 \mu \mathrm{s}$. (All lengths are inferred from full- 
widths-at-half-maxima.) The contraction of the exciuded flux profile, on the other hand, is hardly noticeable with $l_{\min }$ remaining greater than $1.2 \mathrm{~m}$. After the peak contraction $(t>10 \mu \mathrm{s})$ the plasma expands axially with the $\int \mathrm{ndl}(\mathrm{z})$ profile taking the shape of $r_{\Delta \Phi}(z)$ by time $t=20 \mu \mathrm{s}$. Combining the density and magnetic data, one can estimate the particle inventory $\mathrm{N}$. At time $t=15 \mu \mathrm{s}, \mathrm{N}$ is found to be $2.6 \times 10^{20}$ which implies that approximately $70 \%$ of the initial $D_{2}$ fill inside the $\theta$-pinch coil is swept-up during FRC formation.

At larger bias (Figs. 15-18), the formation dynamics are more pronounced. The integral density at axial positions $\mathrm{z}= \pm 0.4 \mathrm{~m}$ is often zero during the peak of the axial contraction. The excluded flux array also indicates stronger contractions although the corresponding $l_{\min }$ values remain consistently $50-100 \%$ larger than those inferred from interferometry (Fig. 19). This discrepancy cannot be explained by the failure of the excluded flux array to accurately infer short FRC lengths. Another noteworthy feature is the $2-4 \mu$ s delay between the density and excluded flux peak contractions. The observed stronger dynamics are illustrated in Figs. 20 and 21 in which maximum excluded flux radii $r_{\max }$, minimum lengths (from interferometry), and minimum elongations $\varepsilon_{\min }=l_{\min } / 2 r_{\max }$ are plotted for a number of discharges obtained over the full range of $B_{b}$ and $\Delta \tau_{P I}$. For a given graph, each data point corresponds to a separate discharge.

The poloidal flux $\Phi_{\mathrm{p}}$ trapped within the FRC can be inferred from the excluded flux, provided one assumes the form of the FRC equilibrium. $\Phi_{p}$ is estimated beginning at time $t=20 \mu \mathrm{s}$, after the formation dynamics subside. A typical, diffuse radial equilibrium profile is assumed (with the parameter $\varepsilon$ defined in Eq. 14 in the paper by Spencer et al., ${ }^{17}$ set equal to -0.25). The corresponding trapped flux can then be written as:

$$
\Phi_{p}=B_{w} r_{w}^{2} x_{s}^{3.25}
$$

where $B_{w}$ is the external $\mathrm{B}$-field, $r_{w}$ is the $\theta$-pinch coil radius, $x_{s}$ is the plasma separatrix radius normalized to $r_{u}$. The flux at time $\mathrm{t}=20 \mu \mathrm{s}$ is found to vary between 2 and $20 \mathrm{mWb}$ (Fig. 22) with the largest values obtained at highest $B_{z c}$ (i.e., at highest bias and with $\Delta \tau_{P I}=5 \mu \mathrm{s}$ ). The inferred $\Phi_{p}$ at $\mathrm{t}=20 \mu$ s ranges between $30 \%$ and $80 \%$ of the magnetic flux at field reversal, $\pi r_{t}^{2} B_{z c}$. 


\section{Equilibrium}

The FRC equilibrium phase following formation begins at about time $\mathrm{t}=20 \mu \mathrm{s}$. Radial equilibrium is maintained over an Alfvén transit time, $2 r_{s} / v_{A}<3 \mu \mathrm{s}$. The inferred radial profile of the integral density varies with $\Phi_{p}$ (or $x_{s}$ ) as shown by the data points in Figs. 23a and 24a for two typical discharges taken at low and high $B_{b}$, respectively. These data have been obtained near the start of the equilibrium phase. A "density hole" is evident at large $x_{s}$, indicative of a more peaked $11(\mathrm{r})$ profile. The dashed lines also plotied in Figs. 23a and 24a are best fits of these data computed assuming the usual ${ }^{18}$ equilibrium profile

$$
n= \begin{cases}n_{m}\left\{1-\left(1-\beta_{s}\right)\left|\frac{2 r^{2}}{r_{2}^{2}}-1\right|^{\eta}\right\} & r \leq r_{s} \\ \beta_{s} n_{m} \exp -\left\{\frac{r^{2}-r_{a}^{2}}{2 r_{s} a}\right\} & r \geq r_{s}\end{cases}
$$

where $\eta$ is an integer between 2 and 5 . The average beta condition, $\langle\beta\rangle=$ $1-1 / 2 x_{9}^{2}$, inplies that there is an additional constraint

$$
P_{s}=1-(\eta+1) x_{s}^{2} / 2
$$

Therefore, the computer fits the six integral density measurements using only three parameters $\mathrm{n}_{m}$, a, and $\eta$. The software accomplishes this by fitting the data with $\mathrm{n}_{m}$ and a for every $\eta$. The best fit (i.e., minimum error) results in the final selection of $\eta$. The corresponding $n(r) / n_{m}$ profiles inferred from the data witt Eqs. (2) and (3) are plotted in Figs. 23b and $24 b$.

At low $x_{s}$ (e.g., see Fig. 23) the inferred $\mathrm{n}(\mathrm{r})$ profiles are somewhat flat $(\eta=3)$ and $\beta_{s}$ is always large $(\geq 0.7)$. The maximum density varies, $2 \times 10^{15}<n_{m}<3 \times 10^{15} \mathrm{~cm}^{-3}$, depending on the time and discharge. The density scale length a outside the separatrix is best estimated later during a discharge when the two outermost interferometer chords view mainly the open-field-line plasma. The scale length varies from shot-to-shot between 0.7 and $2 \mathrm{~cm}$, which corresponds to $2 \rightarrow 6$ ion gyroradii (assuming $\mathrm{T}_{i} \simeq$ $T_{T} / 2=100 \mathrm{eV}$ and $\left.B_{w}=4 \mathrm{kG}\right)$.

At high $x_{s}$ (e.g., see Fig. 24), $\mathrm{n}(\mathrm{r})$ is more peaked near the field null ( $\eta$ $=2$ ). $\beta_{\mathrm{s}}$ is modest initially (typically about 0.4 ) and it usually increases by $50 \%$ during the course of a discharge as $x_{s}$ decreases. The density 
scale length a cannol be accurately determined in this case since all of the interferometers view a substantial part of the closed-field-line region.

During the equilibrium phase the $\int n d l(z)$ and $r_{\Delta \Phi}(z)$ profiles appear somewhat similar (Figs, 25 and 26). The $n=2$ rotational instability is evident at $t \geq 60 \mu \mathrm{s}$ in low- $\Phi_{\mathrm{p}}$, long-lived discharges. From the data in Figs. 25 and 26 one finds that the plasma extends into the mirror regions which begin at $z= \pm 71 \mathrm{~cm}$. One might, therefore, expect the mirror fields to affect $\langle\beta\rangle$, and, consequently, the inferred $\Phi_{p}$. Changes in $\langle\beta\rangle$ due to the mirrors have been estimated using Eq. A3 in the paper by Spencer and Tuszewski. ${ }^{19}$ The FRX-C/LSM plasma separatrix profile is first crudely modeled as an 18 -cm-radius cylinder $\left(x_{s}=0.47\right)$ of variable length with hemispherical ends. The computed $\langle\beta\rangle$ values are plotted in Fig. 27a for plasma lengths $l_{p}$ (defined as the distance between $\mathrm{x}$-points) ranging between 40 and $160 \mathrm{~cm}$. Significant deviations in $\langle\beta\rangle$ from the expected $\left(1-x_{s}^{2} / 2\right)$ value are found for $l_{p} \geq 125 \mathrm{~cm}$. In the experiment, the profile $r_{s}(z)$ is clearly more elliptical; therefore, these rough estimates should be considered as upper bounds. Indeed, for elliptical separatrices, the computad $\langle\beta\rangle$ changes (Fig. 27b) are smaller, although they remain non-negligible for $l_{p} \geq 150 \mathrm{~cm}$. Changes in the inferred $\Phi_{p}$ caused by the mirrors will soon be calculated with 2-D MHD equilibrium computations. ${ }^{20}$

\section{Flux Confinement}

The FRC confinement properties during the equilibrium phase are briefly summarized in this section. We restrict our attention to flux confinement. Particle and energy loss information can be extracted from the data, if desired, in future work. The poloidal flux $\Phi_{p}$ is inferred from external flux measurements and an equilibrium model (see Sec. IV). While a particular $\Phi_{p}$ for a diffuse-profile equilibrium is given by Eq. $1, \Phi_{p}$ values for all equilibria are bounded by two extrema, corresponding to the low and highflux sharp boundary profiles, ${ }^{21}$

$$
\Phi_{L F}=\frac{\pi x_{s}^{2} r_{s}^{2} B_{w}}{4}
$$




$$
\Phi_{H F}=\frac{\pi x_{s}}{2 \sqrt{2}} r_{s}^{2} B_{w}
$$

The time history of $\Phi_{p}$ has been calculated from Eqs. (1) or (4)-(5) for every discharge. The decay of $\Phi_{p}$ for each discharge can be usually classified into one of four modes:

MODE 1: Best $\Phi_{p}$ confinement

MODE 2: Poor $\Phi_{p}$ confinement with relatively uniform decay rate

MODE 3: Poor $\Phi_{p}$ confinement with a faster decay initially

MODE 4: Poor $\Phi_{p}$ confinement with a slower decay initially

Examples of flux decay for each mode are illustrated in Fig. 28. Each graph corresponds to a separate discharge and contains three plots. The broken lines denote the extrema in $\Phi_{p}$ (Eqs. 4 and 5) while the solid lines indicate the flux for the diffuse equilibrium prof:le $\left(E r_{1} .1\right)$. The relative frequencies of occurrence of these modes are displayed in the Fig. 29 histograms.

Mode 1 discharges are defined as those in which the exponential decay time $\tau_{\Phi}=-\Phi /\left(\frac{d \Phi}{d t}\right)$ is greater than several axial Alfvén transit times, i.e., $\tau_{\Phi}>80 \mu \mathrm{s}$. Mode 1 has been obtained only at $\Delta \tau_{P I}=12 \mu \mathrm{s}$ and 0.6 $\leq B_{b} \leq 0.7 \mathrm{kG}$. The zero crossing B-fields and the trapped flux at the start of the equilibriuin phase are relatively small, always less than $0.3 \mathrm{kG}$ and $6 \mathrm{mWb}$, respectively.

Mode 2 discharges are characterized by relatively fast, uniform flux decay, $\tau_{\Phi} \leq 30 \mu \mathrm{s}$. Mode 2 has been observed at all bias fields with $\Delta \tau_{P I}=$ $30 \mu \mathrm{s}$ and also at $B_{b} \leq 0.65 \mathrm{kG}$ or $B_{b} \geq 0.8 \mathrm{kG}$ with $\Delta \tau_{P I}=5$ or $13 \mu \mathrm{sec}$. In Mode 3 discharges, $\tau_{\Phi}$ is again short but the decay of flux is somewhat faster during the first $10-20 \mu$ s of the equilibrium phase. Mode 3 is obtained almost exclusively at higher bias, $B_{b}>0.9 \mathrm{kG}$ on approximately $50 \%$ of the discharges.

Mode 4 discharges are characterized by intermediate confinement tim $=s$ initially, $\tau_{\Phi} \simeq 50 \mu \mathrm{s}$, followed by a rapid loss in flux at later times $\mathrm{t}>30 \mu \mathrm{s}$. The rapid flux loss is sometimes (but not always) accompanied by an axial shift of the excluded flux radius center of volume. Mode 4 is obtained primarily at $\Delta \tau_{P I}=5 \mu$ s with modest bias fields $B_{b}=0.65 \rightarrow 0.9 \mathrm{kG}$. 


\section{Discussion}

In the FRX-C/LSM device, it has been demonstrated that the preionization and reversed bias field strengths clearly influence the FRC formation dynamics and the equilibrium confinement.

At low bias strengths the magnetic field inside the $\theta$-pinch coil is initially reversed by the PI-bank discharge and the $D_{2}$ fill breaks down just after the second B-field zero-crossing. The breakdown begins at a time when $\mathrm{B}$ is smaller than the applied bias field $B_{b}$. Therefore, to maximize flux trapping, the applied bias nust diffuse tinrough the PI plasma. Irternal magnetic field measurements are necessary to determine the amount of diffusion. Unfortunately, such measurements have not yet been attempted in FRX-C/LSM. There is, however, possible indirect evidence of fast field diffusion during the early stages of the PI. At $\Delta \tau_{P I}=5 \mu \mathrm{s}$, the external Bfield from the PI capacitor bank adds to the bias at the time when the main bank is discharged. The diffusion of this added bias might be responsible for the observed increased trapped flux at field reversal $\pi B_{z c} r_{t}^{2}$.

The PI plasma is clearly more dynamic at lower bias fields (Figs. 5 and 6). Radial compression and axial density waves are observed. At larger bias, breakdown occurs more gradually (Figs. 7 and 8) and the piasma appears stiffened by the larger fields. A transition field is found above which the radial and axial structure in PI plasma density becomes much less pronounced. This transition occurs at about $1.1 \mathrm{kG}$, a value considerably larger than the bias, $B_{b}=0.8 \mathrm{kG}$, at which the $\theta$-pinch PI B-field grazes zero.

As the bias field strength is increased, more flux is trapped at each $\Delta \tau_{P I}$ setting (Fig. 22). The $\theta$-pinch-PI breakdown occurs without zero crossing but usually not at the maximum $E_{\theta}$. Larger bias results in more severe axial contractions and larger plasma radii during FRC formation as illustrated in Fig. 20. The decrease in minimum elongation $\varepsilon_{\min }$ with increased $B_{z c}$ is in qualitative agreement with earlier experiments ${ }^{10}$ and theory. ${ }^{9}$ An interesting observation is that the plasma contraction is faster and stronger than that of the excluded flux profile (Fig. 18). Barring any unknown systematic errors in the measurements, this effect appears to imply non-negligible contributions of plasma inertia during formation. 
Indeed, the MHD simulations with the Milroy-Brackbill code support this hypothesis The simulations show that the minimum length $\ell_{\min }$ as inferred from the de: $:$ iy measurement is indeed shorter than that inferred from the excluded flux measurement. The results from a typical simulation are presented in Fig. 30 which shows the variation of the full-width-at-halfmaximum FRC length as inferred using a) the excluded flux radius, b) the $\int \mathrm{ndl}$ signal, and c) the line density. It is seen that the length estimated using the excluded flux radius is always larger than the length estimated from $\int \mathrm{ndl}$ and from the line density. This is expected because only the outer flux surfaces extend axially to the FRC ends and the number density on these surfaces is smaller than that on the inner surfaces. Note that the length as estimated using the $\int \mathrm{ndl}$ signal exhibits rapid oscillations for $\mathrm{t} \leq 6 \mu \mathrm{s}$. These oscillations are not caused by axial motion; they occur because the plasma undergoes radial oscillations with different frequencies at different axial locations. It also is seen from the figure that the discrepancy between FRC length as inferred from the excluded flux radius and from the $\int \mathrm{ndl}$ signal is more apparent during the peak axial contraction when the area waves propagating from the FRC ends merge. Figure 31 shows the flux surfaces and the density contours at $\mathrm{t}=16 \mu \mathrm{s}$. It is seen that the density is not constant along the field lines and that it increases towards the $\mathrm{z}=0$ plane.

In FRX-C/LSM, $\varepsilon_{\text {min }}$ typically ranges between 1 and 3 (Fig. 21). Therefore, almost all of our data fall short of the threshold condition, $\varepsilon_{\min } \geq 3$, found necessary for good confinement in FRX-C experiments with the smaller coil and discharge tube. ${ }^{10,11}$ In FRX-C/LSM the best flux confinement to date $\left(\tau_{\Phi}=100 \rightarrow 200 \mu \mathrm{s}\right)$ has been obtained at relatively low flux, $\Phi_{p}<6 \mathrm{mWb}$, and largest minimum elongations $\varepsilon_{\min } \simeq 2.7$. For these plasmas the field null resistivity $\eta$ (inferred assuming a rigid-rotor equilibrium with $T_{e} \simeq T_{T} / 2 \simeq 100 \mathrm{eV}$ ) is at least ten times the classical value with $Z_{e f f}=1$. In earlier FRX-C discharges at similar $T_{e}$ but with $\varepsilon_{\min } \geq 3$, the resistivity anomaly factor was about five for discharges with the best confinement.

FRC formation using the programmed-mode (i.e., with no tearing reconnection) has been demonstrated to be superior ${ }^{1,3}$ in that the strong axial dynamics with $\varepsilon_{\min }<3$ can be tolerated with no subsequent degradation 
in confinement. In fact, it has been reported that $\tau_{\varphi}$ improves as $\Phi_{p}$ is increased. ${ }^{22}$ These observations lead one to anticipate that on upcoming programmed formation experiments on FRX-C/LSM, not only will the confinement improve at low bias but it will improve even further as $B_{b}$ (and $\Phi_{p}$ ) is increased.

\section{Acknowledgments}

The authors thank the technicians from the Los Alarios National Laboratory CTR-3 group for their outstanding technical support during the course of these experinents. The rf pre-preionization system was originally developed by Dr. S. O. Knox (CTR-5) and implemented onto FRX-C/LSM by Professor H. Tuczek (Essen Univ.) and Rita Gribble (CTR-3). Additional optics for the radial interferometer array was designed and installed by Dr. S. Okada (Osaka Univ.). Computer support for our data analyses was developed by P. L. Klingner (CTR-5). Useful technical comments concerning these data from Dr. R. K. Linford (CTR-DO), from Dr. M. Tuszewski (CTR-3, on leave at Fontenay-aux-Roses) and Dr. R. E. Chrien (CTR-3, on leave at Osaka Univ.) are gratefully acknowledged. 


\section{References}

${ }^{1}$ V. V. Belikov, V. M. Goloviznin, V. K. Korshunov, A. P. Kreshchuk, R. Kh. Kurtmullaev, Ya. N. Laukhin, A. I. Malyutin, A. P. Proshletsov, O. L. Rostovtsev, V. N. Semenov, and V. F. Strizhov. Suppression of losses in a compact torus with programmed shaping of the magnetic structure. In Plasma Physics and Controlled Nuclear Fusion 1982, page 343, IAEA, Vienna, 1983. Vol. II (Baltimace conference).

${ }^{2}$ W. T. Armstrong, D. G. Harding, E. A. Crawford, and A. L. Hoffman. Flux-trapping during formation of FRC's. Physics of Fluids, 25:2121, 1982.

${ }^{3}$ J. T. Slough, R. D. Milroy, D. G. Hardirg, and A. L. Hoffman. Experimental and numerical study of various methods of programmed formation. In 7th Compact Torus Symposium, Los Alamos National Laboratory, Los Alamos, NM, 1985. To be published in proceedings of̂ that ineeting.

${ }^{4}$ C. Wu, Y. Aso, S. Himeno, S. Yamaguchi, M. Okamoto, and K. Hirano. Oscillations of the trapped flux in the early phase of a field-reversed $\theta$-pincl. Journal of the Physical Society of Japan, 52:1215, 1983.

${ }^{5} \mathrm{~L}$. C. Steinhauer. Plasma heating in field-reversed $\theta$-pinches. Physics of Fluids, 26:254, 1983.

${ }^{6}$ M. I. Kutuzov, V. N. Semenov, and V. G. Strizhov. Trapping of magnetic flux during the formation of a compact toroid. Sov. J. Plasma Physics, 7:520, 1981.

${ }^{7} \mathrm{G}$. E. Vekshtein. Loss of magnetic flux during the formation of a FRC. Sov. Phys. Dokl., 28:569, 1933.

${ }^{8}$ R. D. Milroy, J. T. Slough, and A. L. Hoffman. Plasma wall sheath contributions to flux retention during the formation of FRC's. Physics of Fluids, 27:1545, 1984.

${ }^{9}$ L. C. Steinhauer. Magnetic flux trapping during field-reversal in the formation of a FRC. Physics of Fluids, 28:3333, 1985. 
${ }^{10} \mathrm{M}$. Tuszewski. Axial dynamics in field-reversed $\theta$-pinches. In 7th Compact Torus Symposium, Los Alamos National Laboratory, Los Alamos, NM, 1985. To be published in proceedings of that meeting.

${ }^{11} \mathrm{~W}$. T. Armstrong. FRC formation studies with $z$-discharge ionization on FRX-C. In 7th Compact Torus Symposium, Los Alamos National Laboratory, Los Alamos, NM, 1985. To be published in proceedings of that meeting.

${ }^{12}$ M. Tuszewski, R. E. Chrien, and E. J. Yavornik. The FRX-C large source modification. In 7th Compact Torus Symposium, Los Alamos National Laboratory, Los Alamos, NM, 1985. To be published in proceedings of that meeting.

${ }^{13}$ R. E. Chrien, M. Tuszewski, and E. J. Yavornik. The FRX-C large source modification. In Proceedings of the 7th US-Japan Workshop on Compact Toroid Research, page 33, Spectra Technology Inc., Bellevue, Washington, 1986.

${ }^{14}$ T. S. Green and A. A. Newton. Diffusion of antiparallel bias B-field during the initial stages of a $\theta$-pinch. Physics of Fluids, 9:1386, 1906.

${ }^{15}$ W. I. Armstrong, J. C. Cochrane, R. J. Commisso, J. Lipson, and M. Tuszewski. $\theta$-pinch ionization for FRC formation. Applied Physics Letters, 38:680, 1981.

${ }^{16}$ R. D. Milroy and J. U. Brackbill. Numerical studies of a FRC plasma. Physics of Fluids, 25:775, 1982.

${ }^{17}$ R. L. Spencer, M. Tuszewski, and R. K. Linford. Adiabatir. compression of elongated FRC's. Physics of Fluids, 26:1564, 1983.

${ }^{18} \mathrm{M}$. Tuszewski. Experimental study of the equilibriun of FRC's. Plasma Phys. and Contro. Fusion, 26:991, 1984.

${ }^{19}$ R. L. Spencer and M. Tuszewski. Experimental and computational equilibria of FRC's. Physics of Fluids, 28:1810, 1985. 
${ }^{20}$ R. B. Webster and J. L. Schwarzmeier. Another FRC equilibrium code. In Proceedings 1980 Sherwood Theory Conf., Courant Inst., New York, 1986. Paper 2C-12.

${ }^{21}$ W. T. Armstrong, R. K. Linford, J. Lipson, D. A. Platts, and E. G. Sherwood. Field-reversed experiments on compact toroids. Physics of Fluids, 24:2068, 1981.

${ }^{22}$ J. T. Slough, A. L. Hoffman, R. D. Milroy, D. G. Harding, and L. C. Steinhauer. Flux and particle lifetimes measurements in FRC's. Nuclear Fusion, 24:1537, 1984. 


\section{FIGURES}



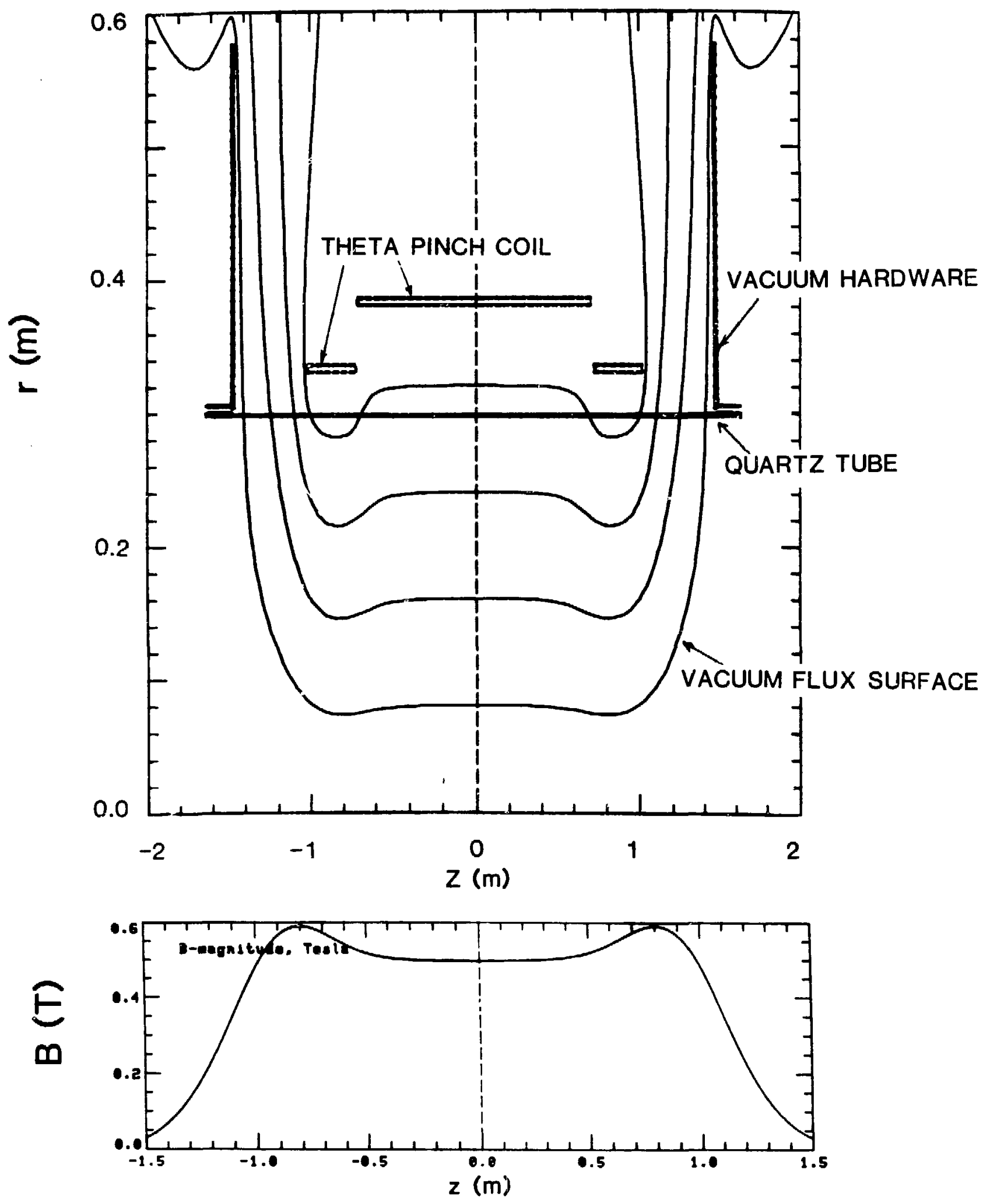

FIG. 1. Schematic diagram of FRX-C/LSM showing sample vacuum magnetic flux surfaces and $B_{z}(z)$-field on axis. 


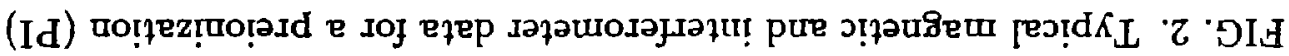
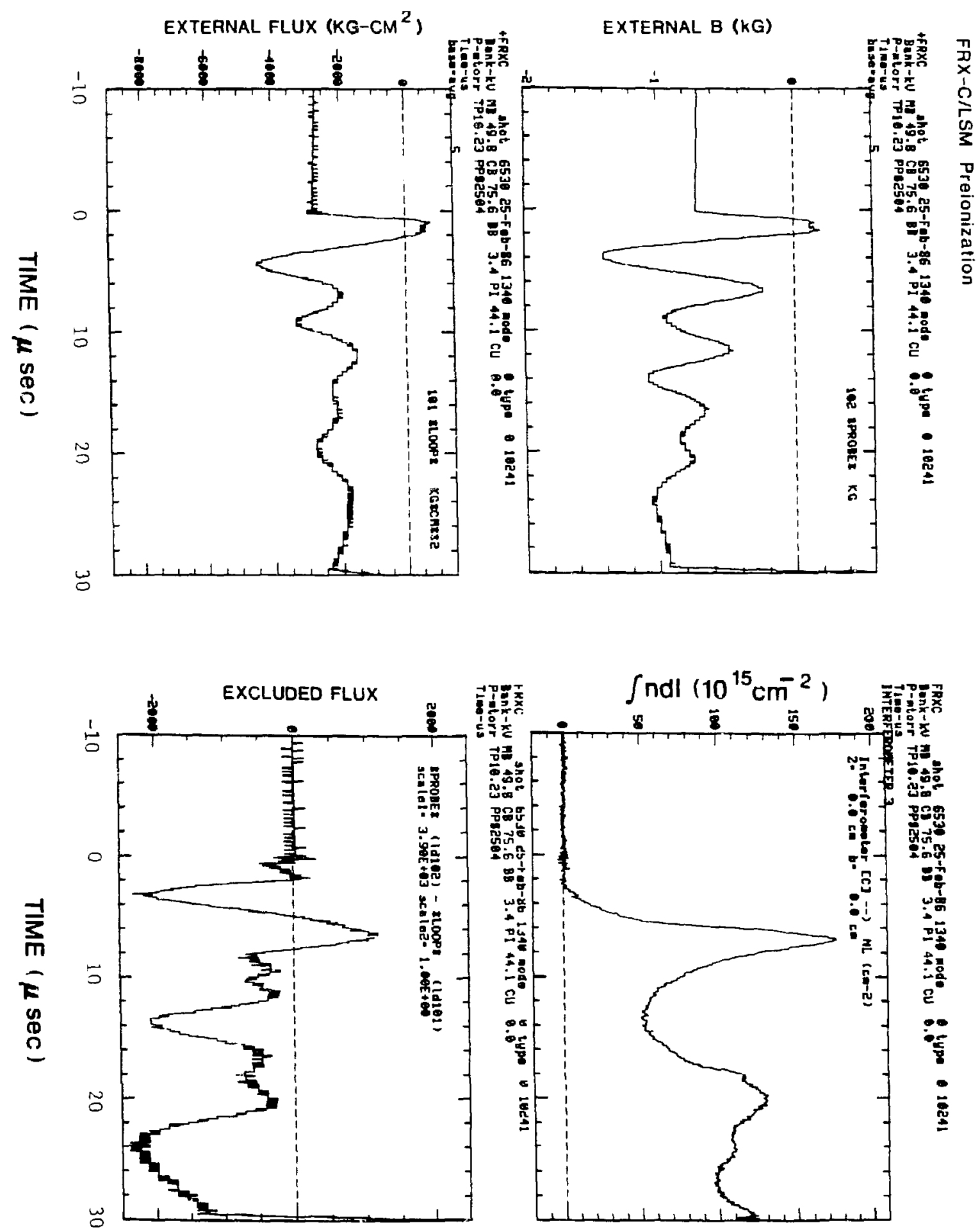


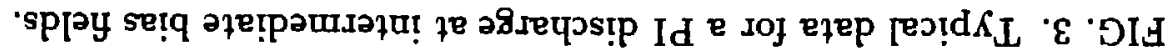
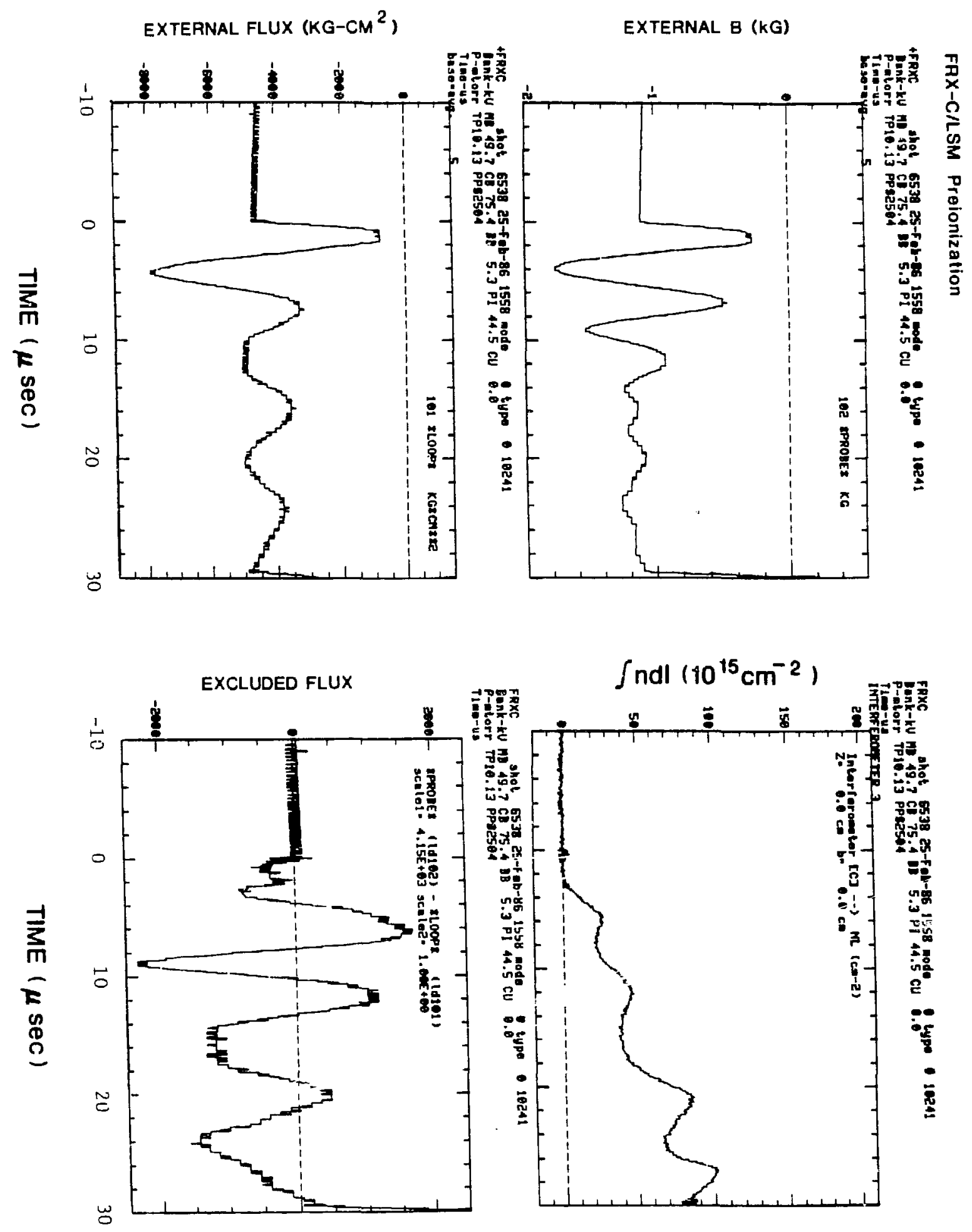


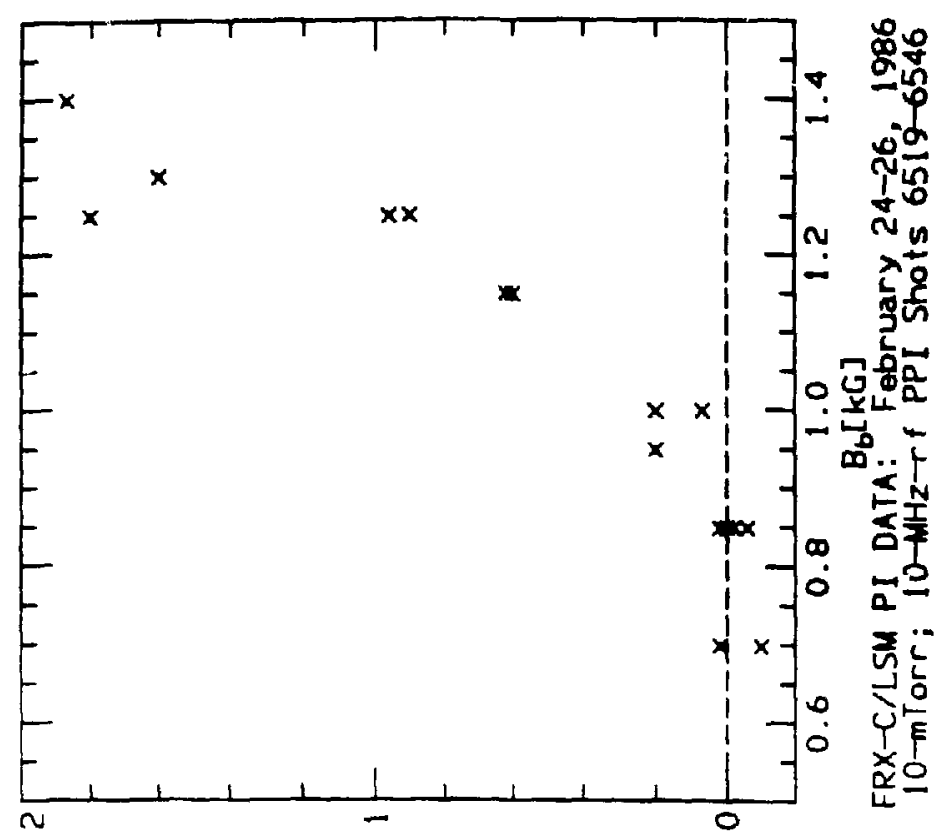

(Dx)

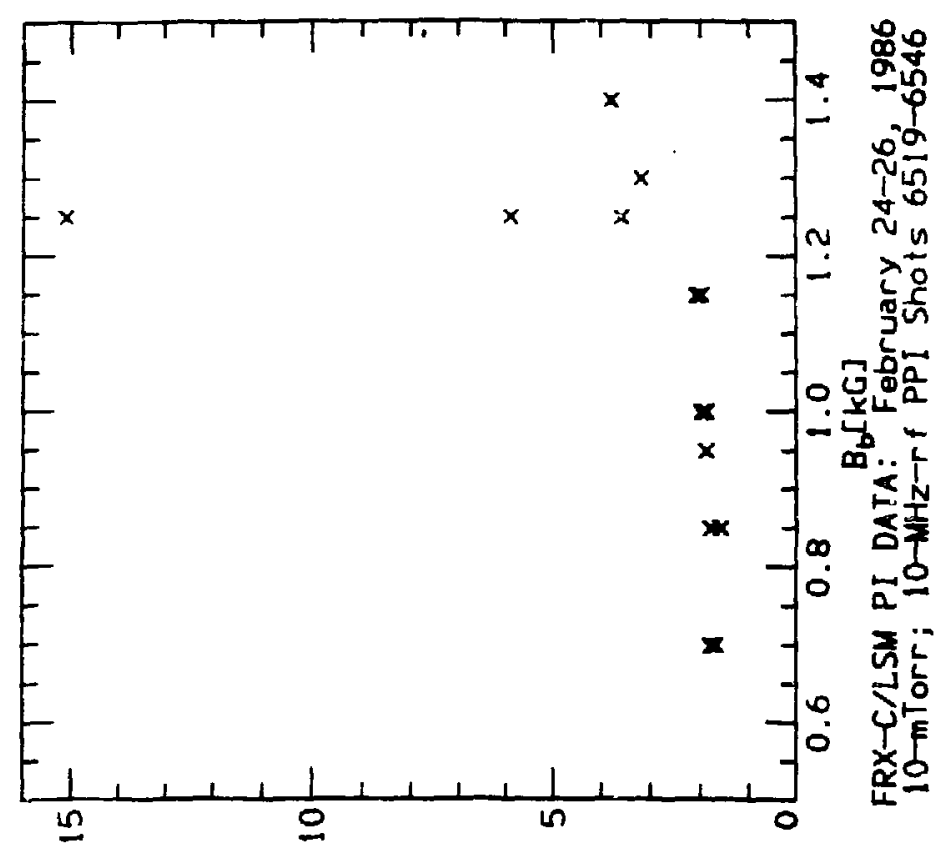

(Sri) mopyosedq

FIG. 4. Time into the PI bank discharge at which first plasma diamagnetism appears plotted against bias field. The magnetic field measured at this time is also plotted. 


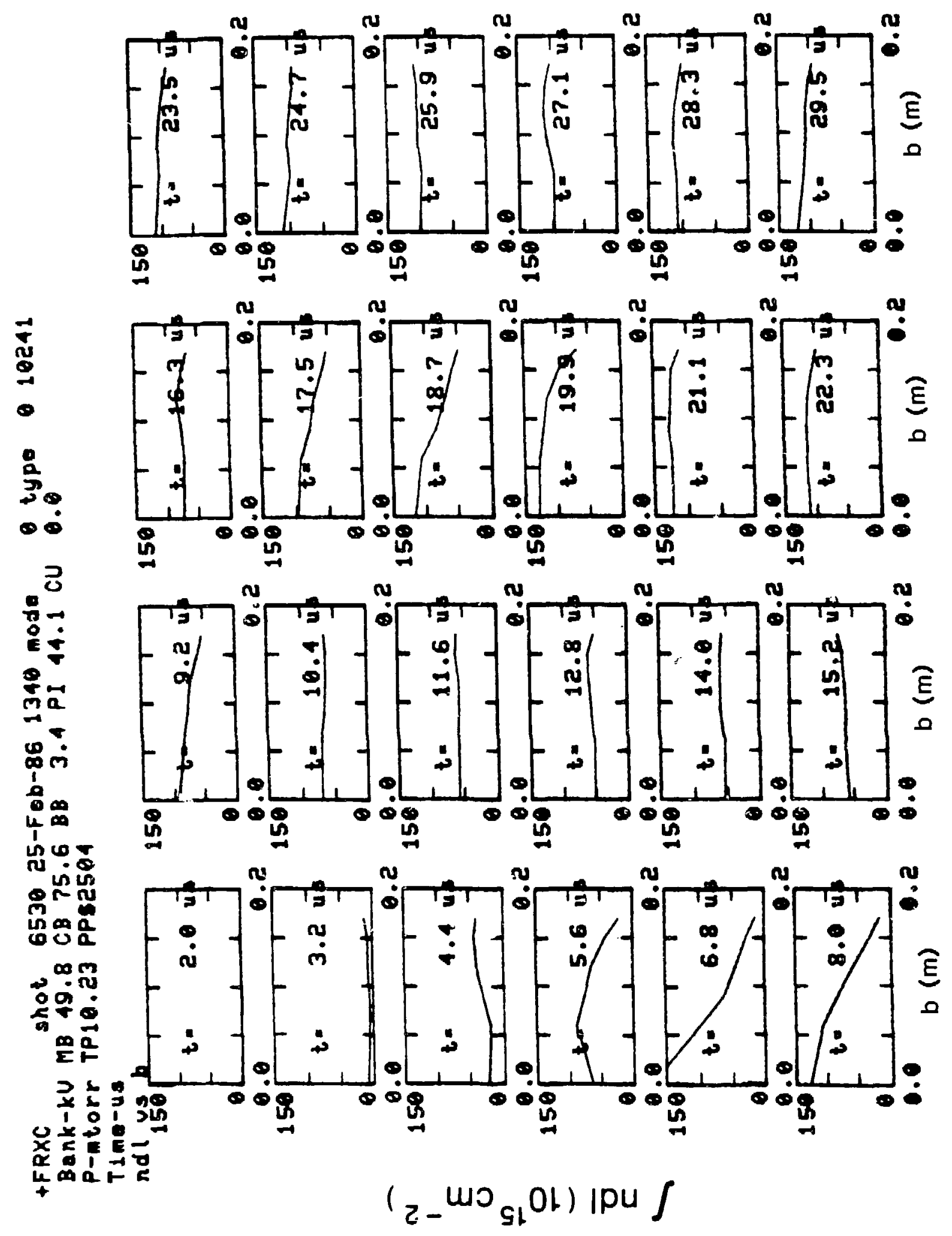

FIG. 5. Time evolution of the radial integral density profile for a PI discharge with low bias field, $B_{b} \simeq 0.75 \mathrm{kG}$. 


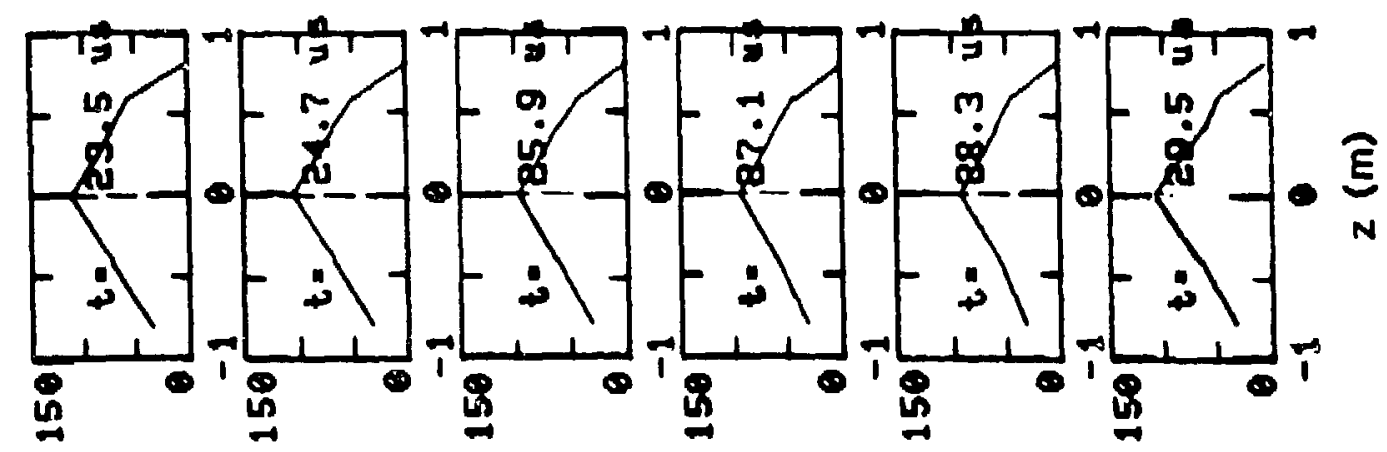

ํㅣㅁ
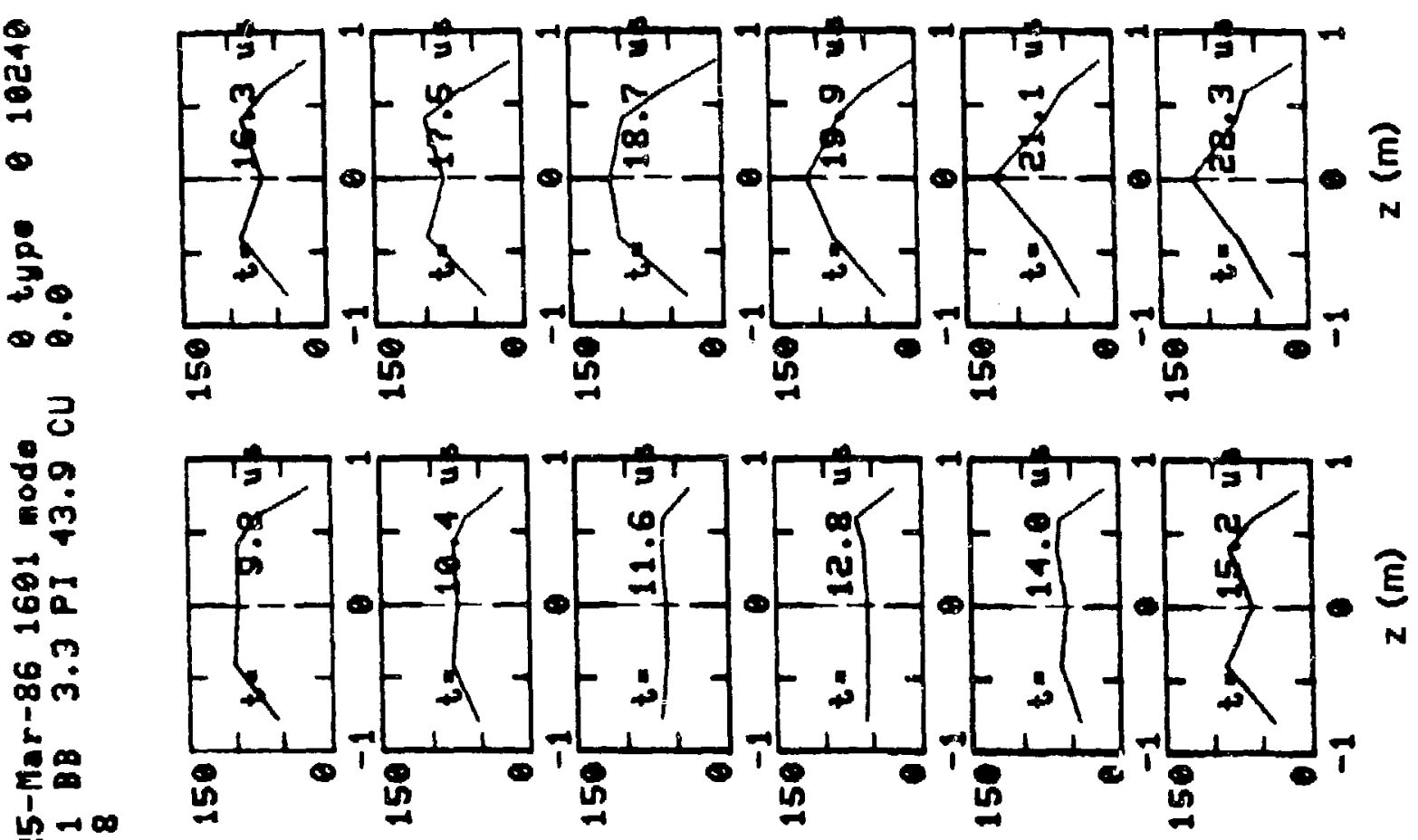

$1 \rightarrow \infty$

a -1

$-40$

a

(1)

600

$r$

Pै:

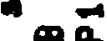

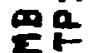

I \& 9 ne

10154

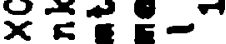
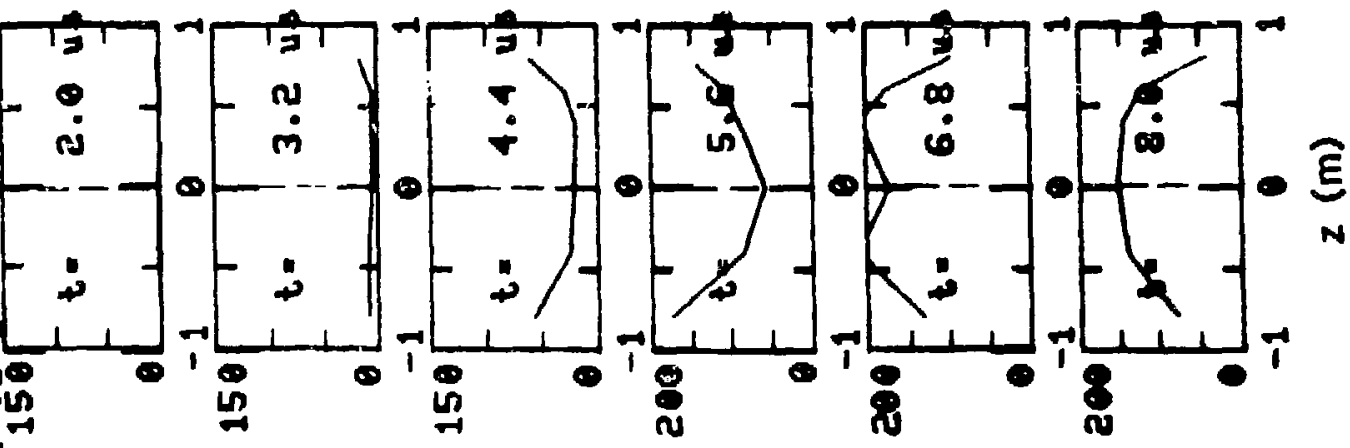

$0<1=0$

Laten

$$
\left(z-w_{\mathrm{sl}} \mathrm{Ot}\right) \mathrm{IPU} \int
$$

FIG. 6. Time evolution of the axial integral density profile for a PI discharge with low bias, $B_{b} \simeq 0.75 \mathrm{kG}$. 


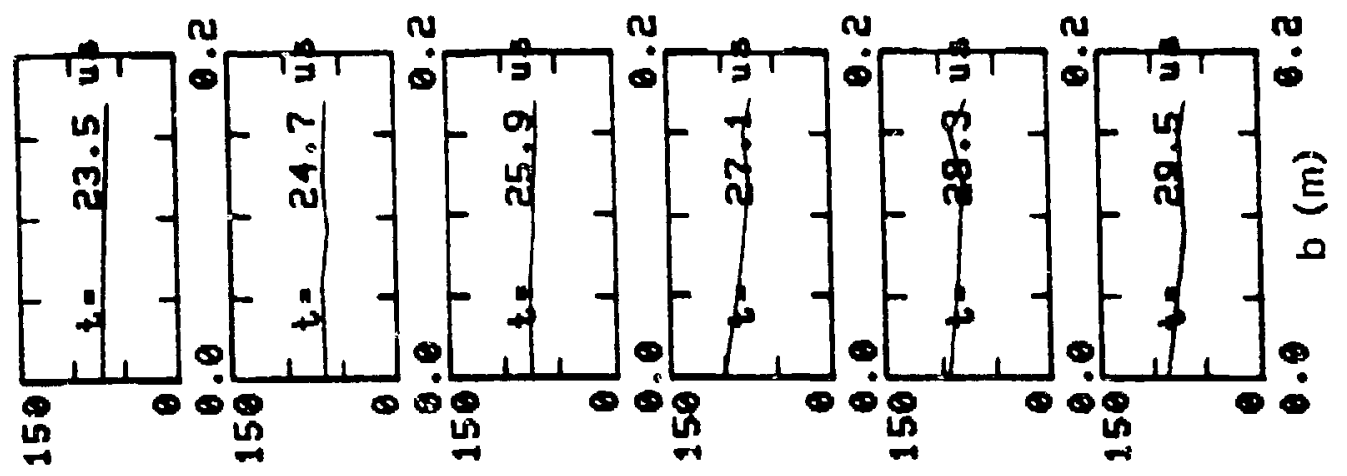

:
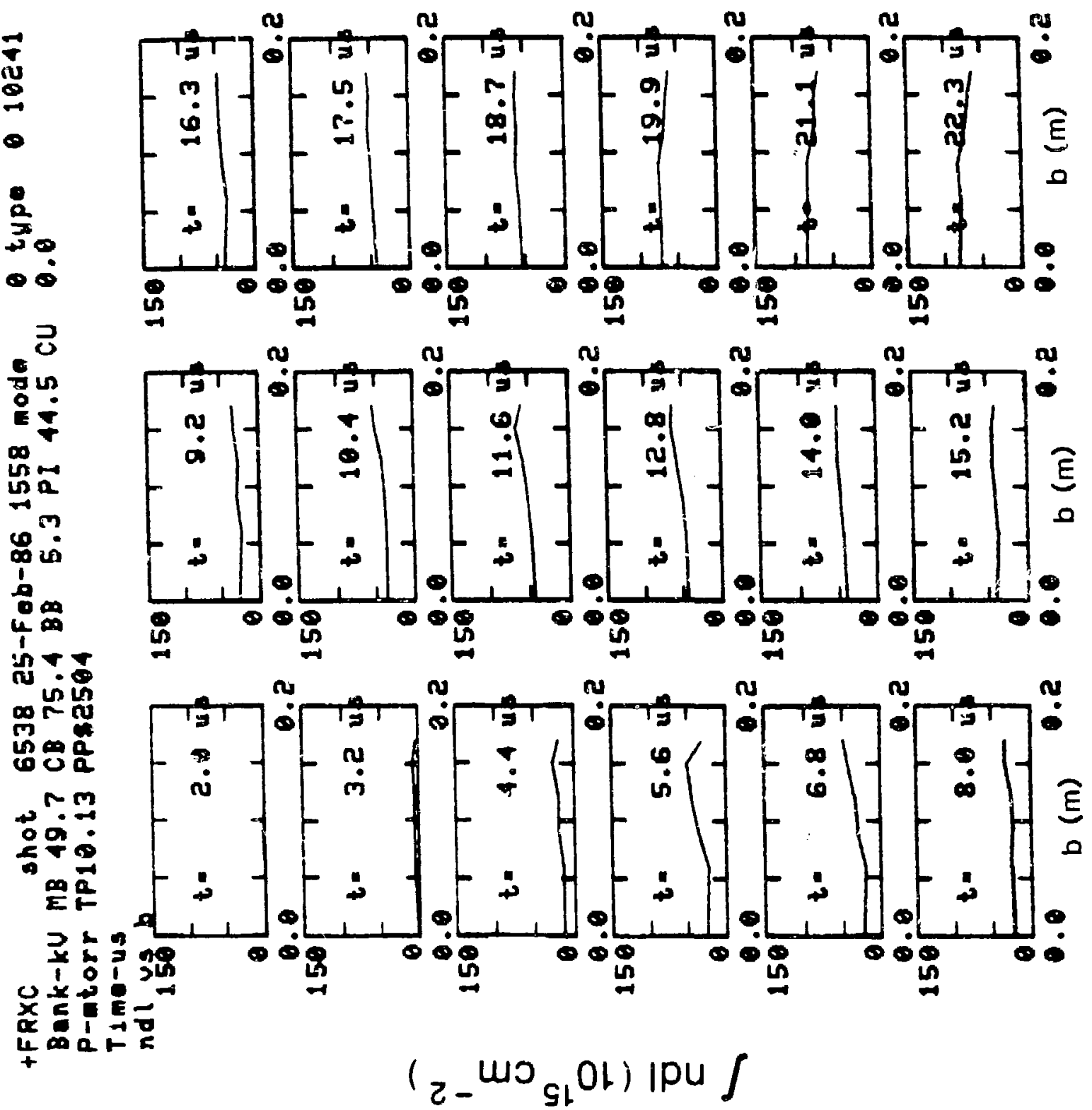

FIG. 7. Time evolution of the radial integral density profile for a PI discharge with intermediate bias, $B_{b}=1.15 \mathrm{kG}$. 


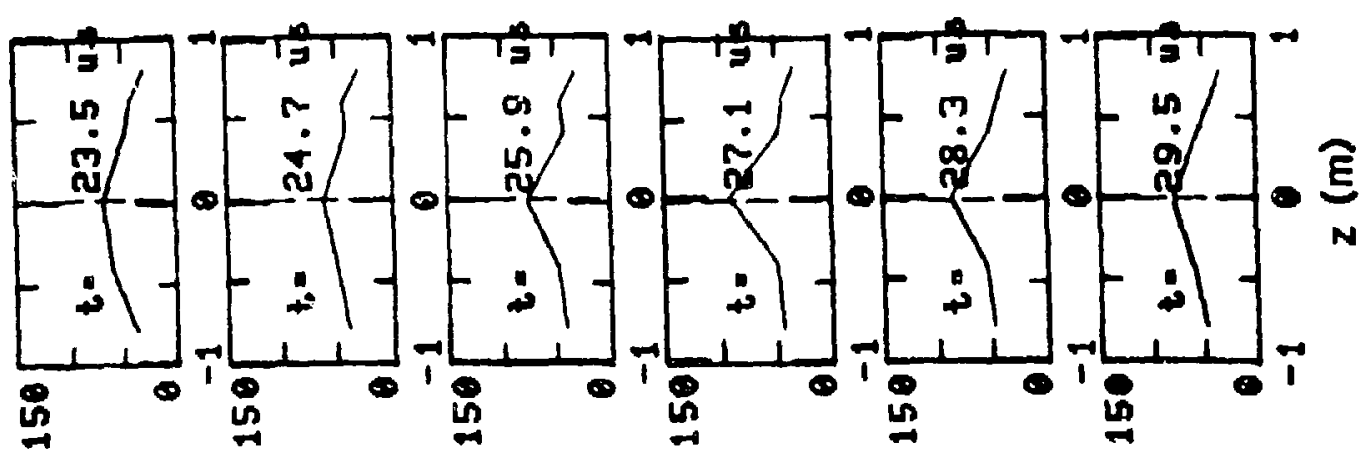

$\frac{2}{2}$

3

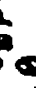

$\infty 0^{\circ}$

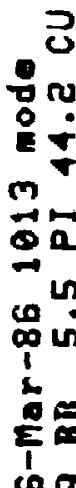
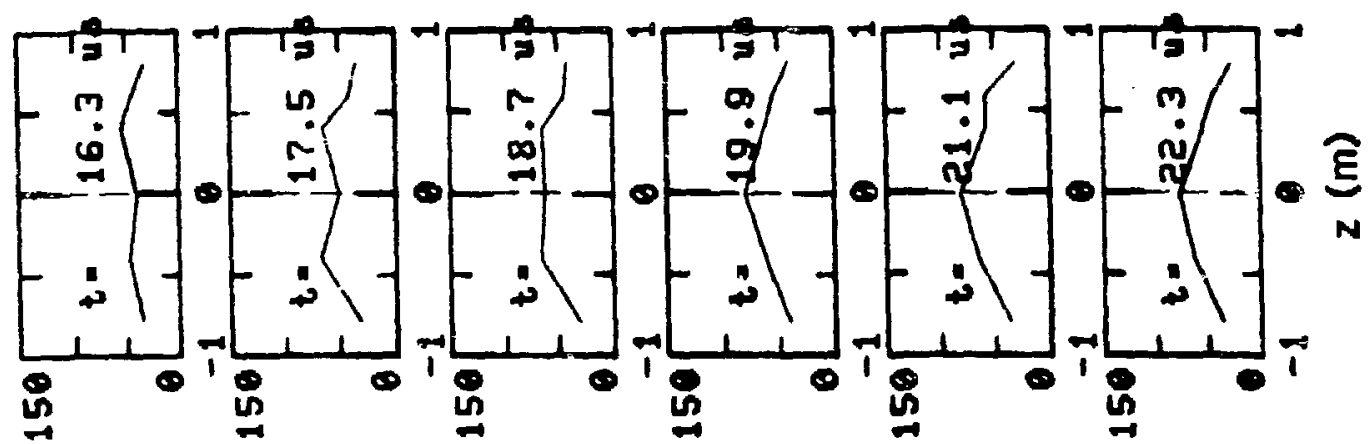

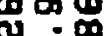

$+7$

cru

con

$\infty 03 \frac{1}{0}$

ro
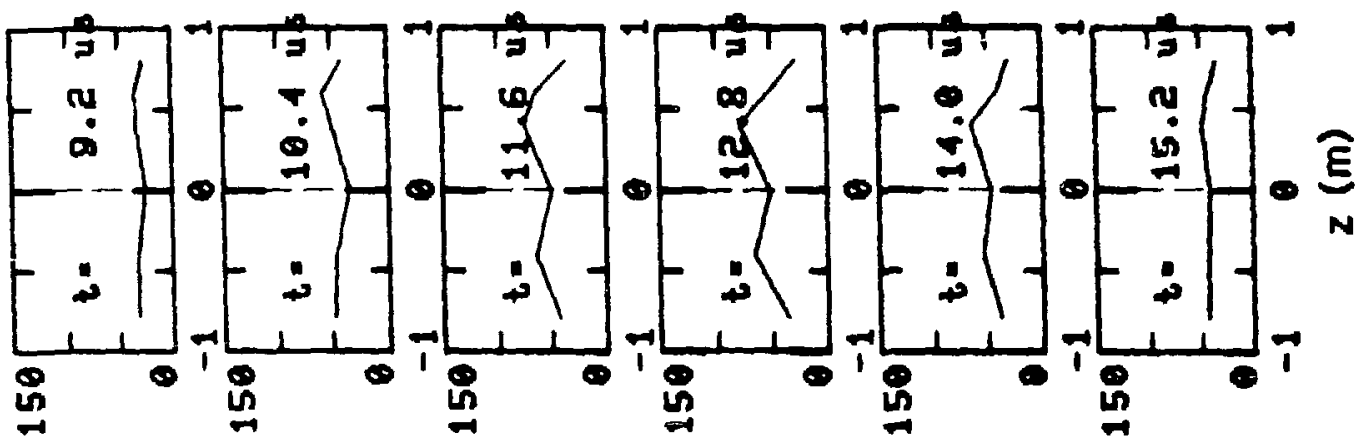

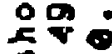

$5 \div$

$\infty$

$3+9$

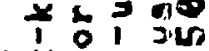

Q

$\times 5 \div=0$

Ita

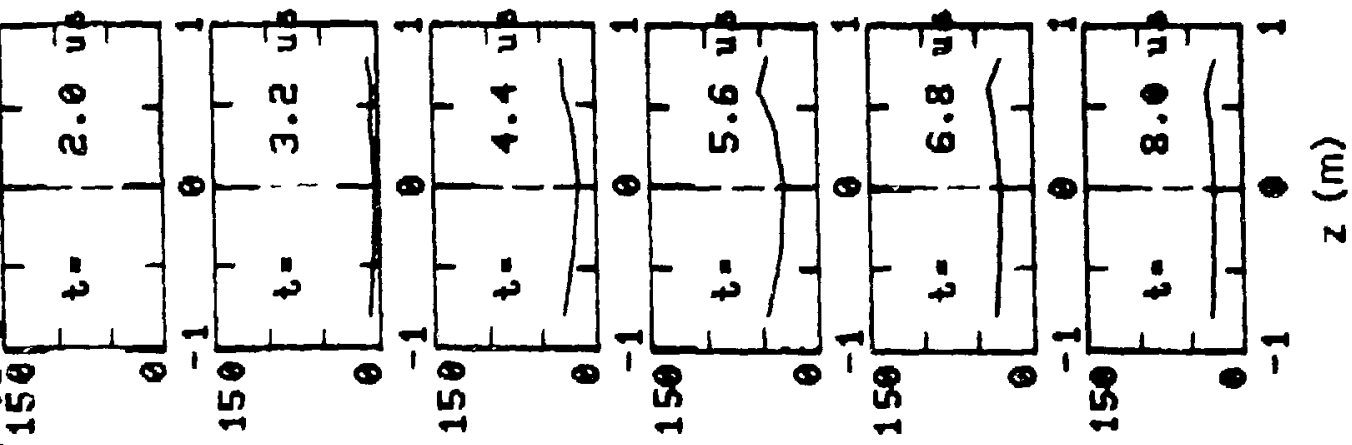

$$
\left(z^{-U O_{s l}} 0 l\right) \text { IPU }
$$

FIG 8. Time evolution of the axial integral density profile for a PI discharge with intermediate bias. $B_{b}=1.15 \mathrm{kG}$. 


\section{velocity field:}
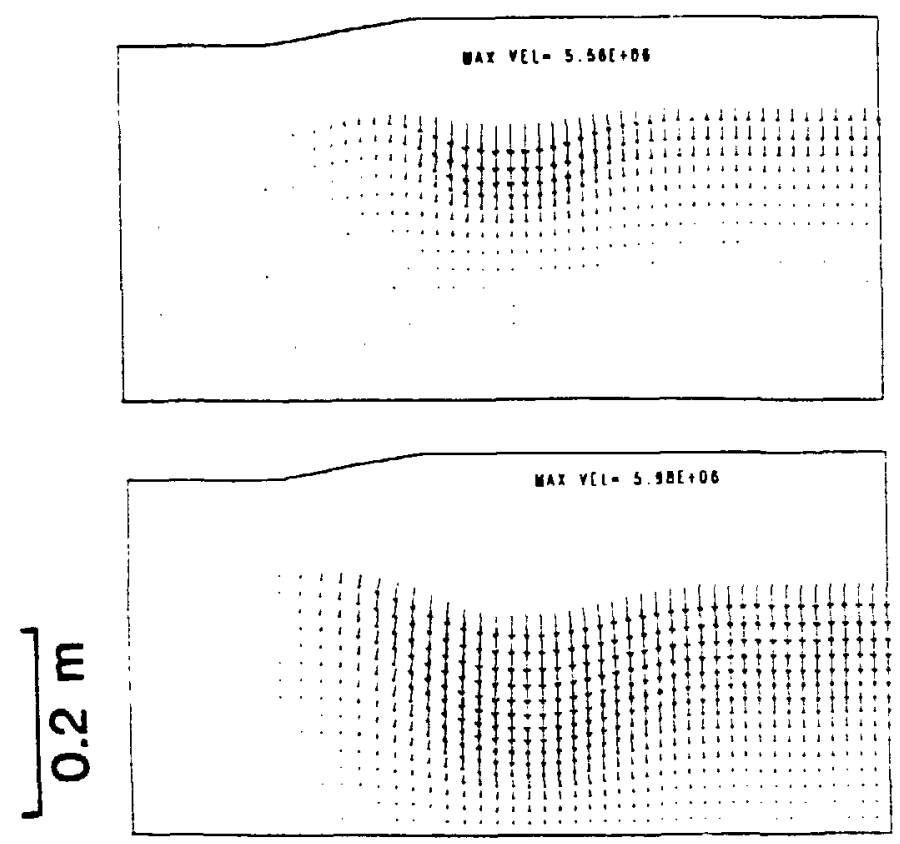

ᄂ

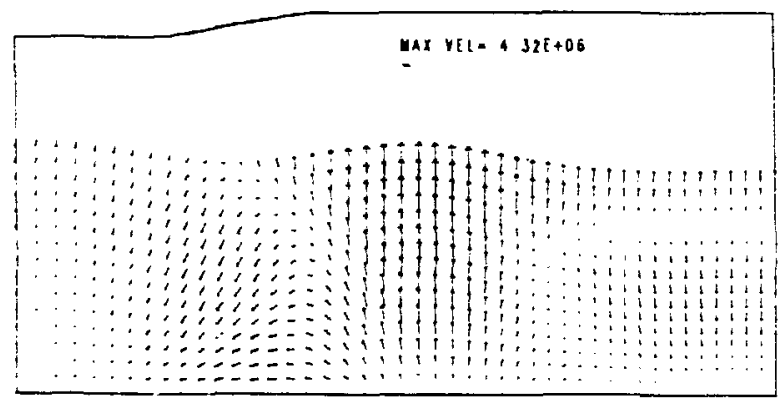

UAX VIL $-6071+06$

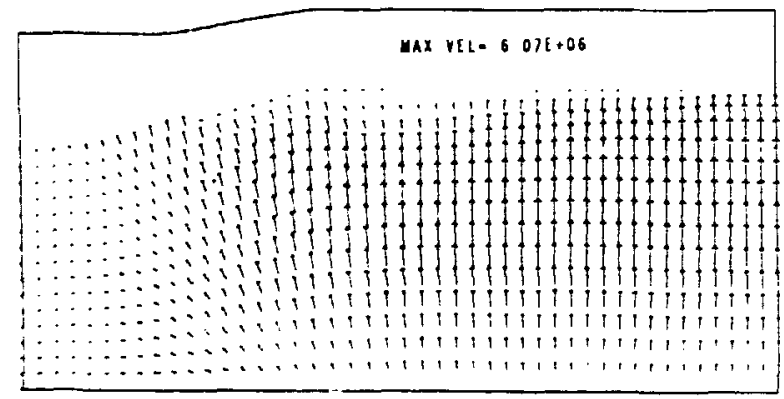

\section{flux contours:}
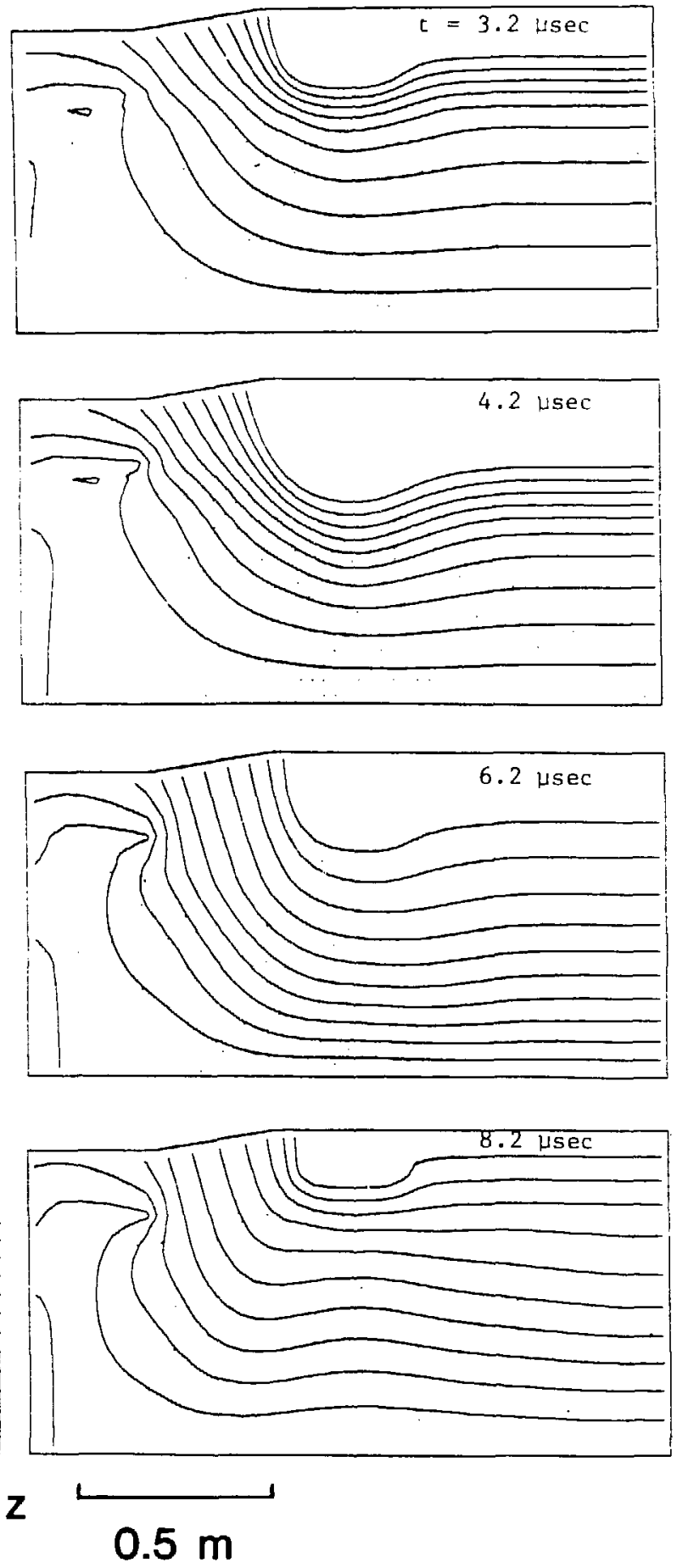

FIG. 9a. I'he results of a numerical simulation of the preionization plasma in LSM using the Milroy-Brackbill code. The velocity vectors and the magnetic flux contours for $\mathrm{t}=3.2,4.2,6.2,8.2,10.2,12.2$, and $14.2 \mu \mathrm{s}$. 


\section{velocity field:}

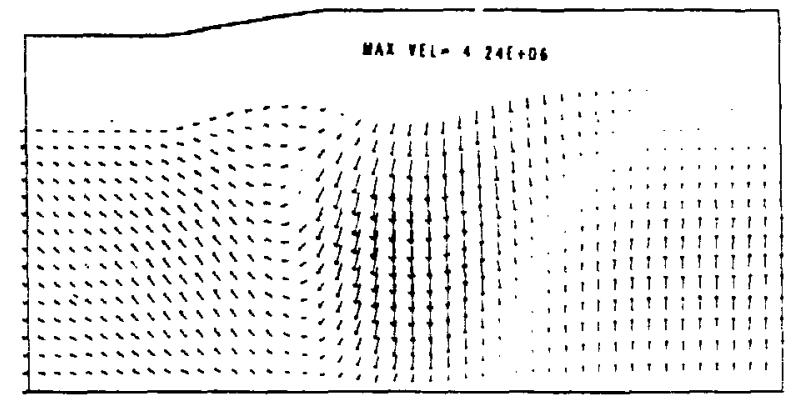

]$_{\text {¿ }}^{\text {E }}$
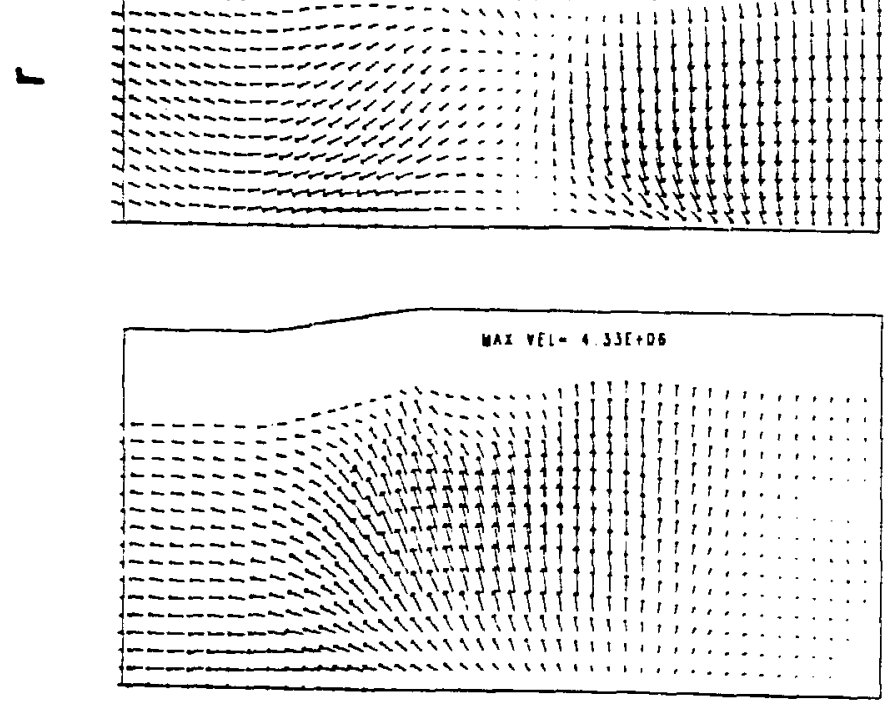

\section{flux contours:}
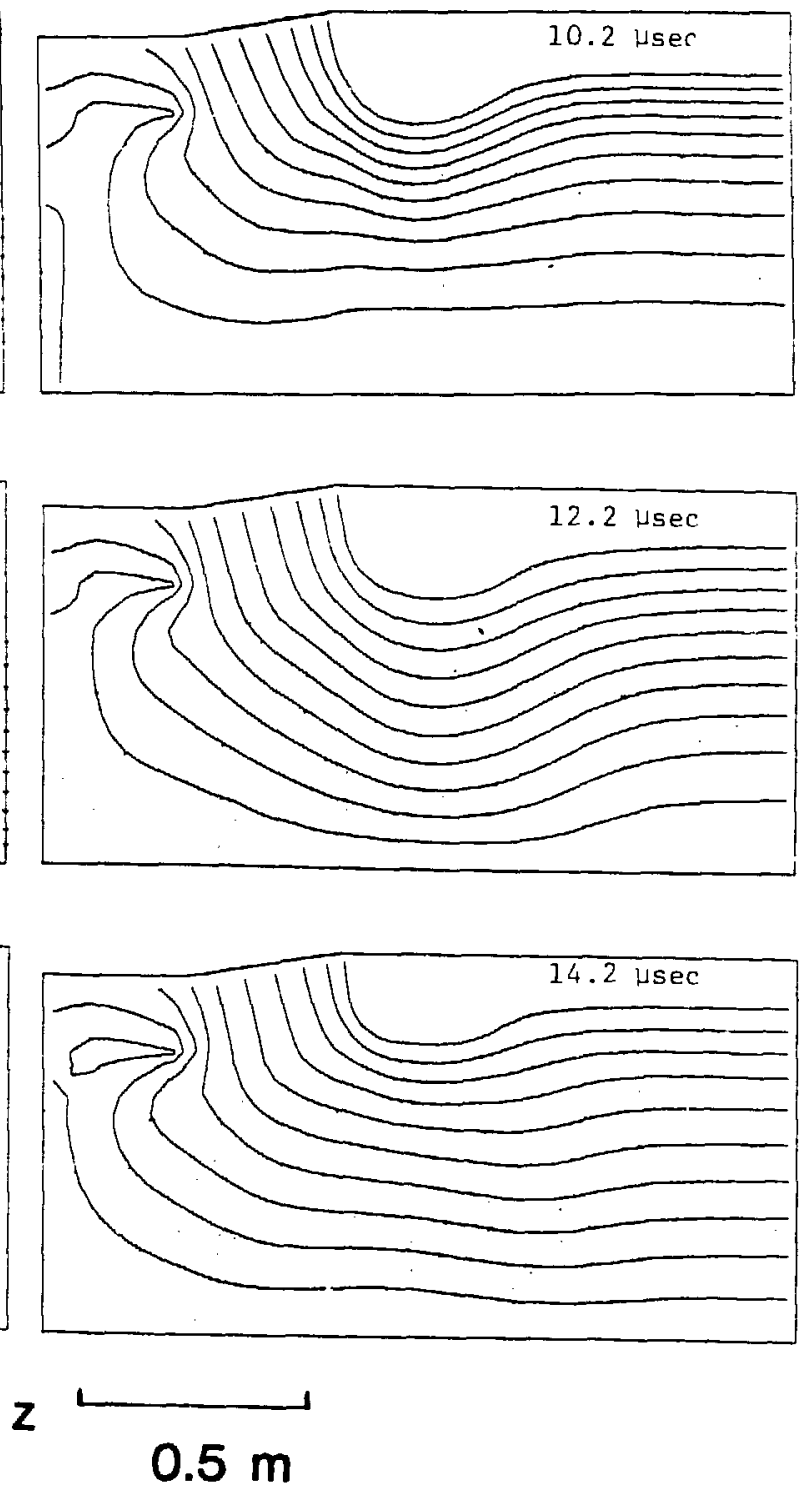

FIG. 9a. (cont) 

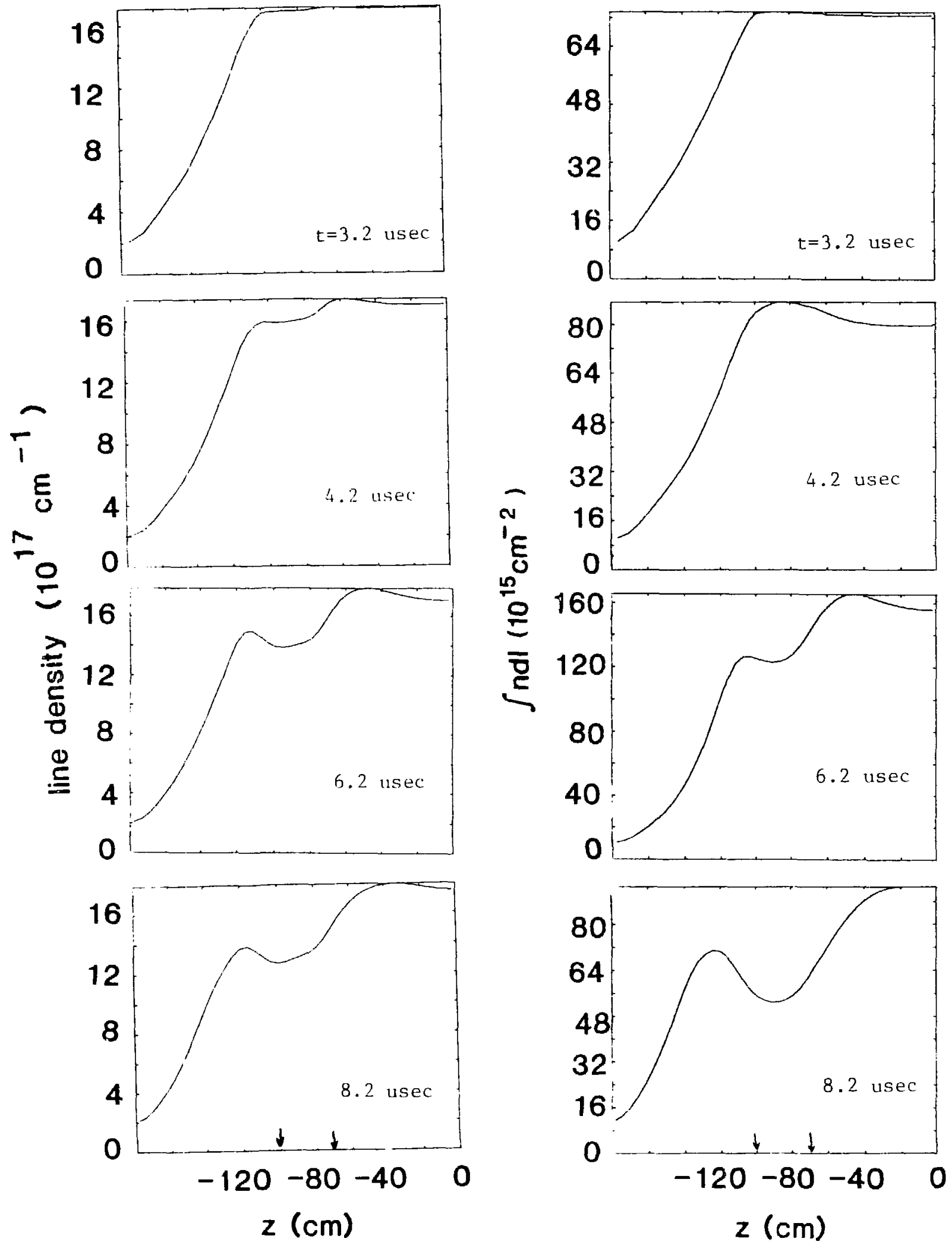

FIG $9 \mathrm{~b}$. The results of a numerical simulation of the preionization plasma in LSM using the Milroy-Brackbill code. Axial profiles of the line density and line integrated density $\left(\int \mathrm{ndl}\right)$ for $\mathrm{t}=3.2,4.2,6.2,8.2,10.2,12.2$, and $14.2 \mu \mathrm{s}$. 


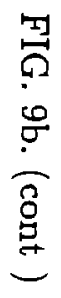

line density $\left(10^{17} \mathrm{~cm}^{-1}\right)$

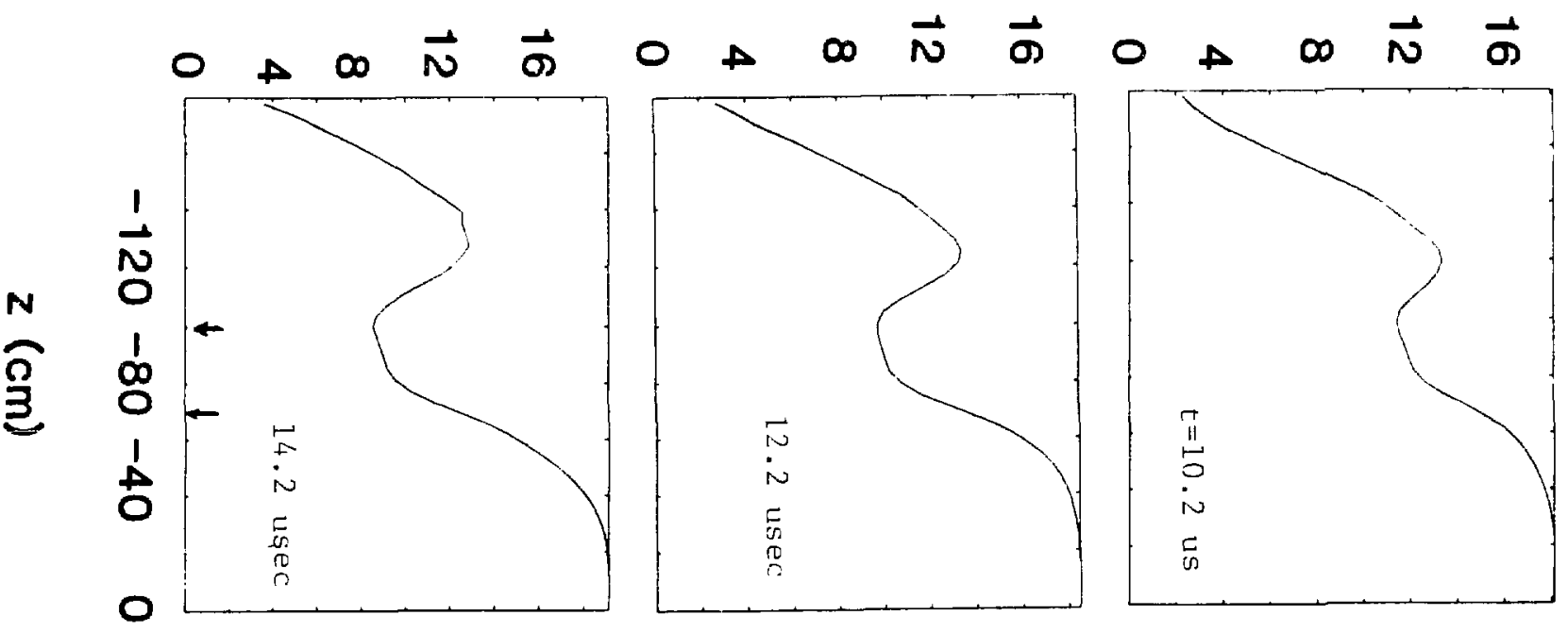

$\int \mathrm{ndl}\left(10^{15} \mathrm{~cm}^{-2}\right)$
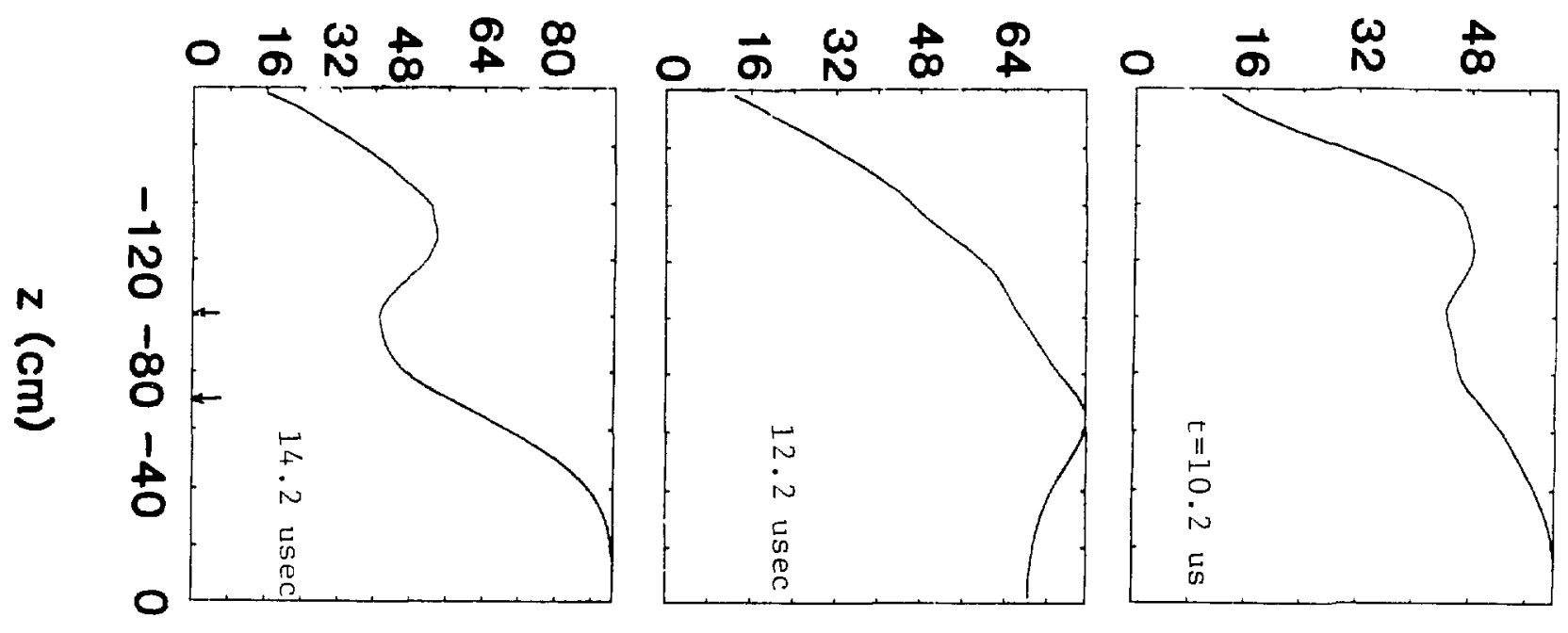


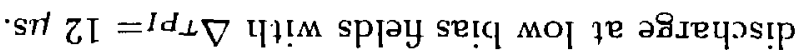

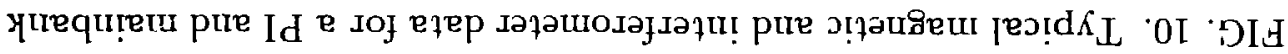
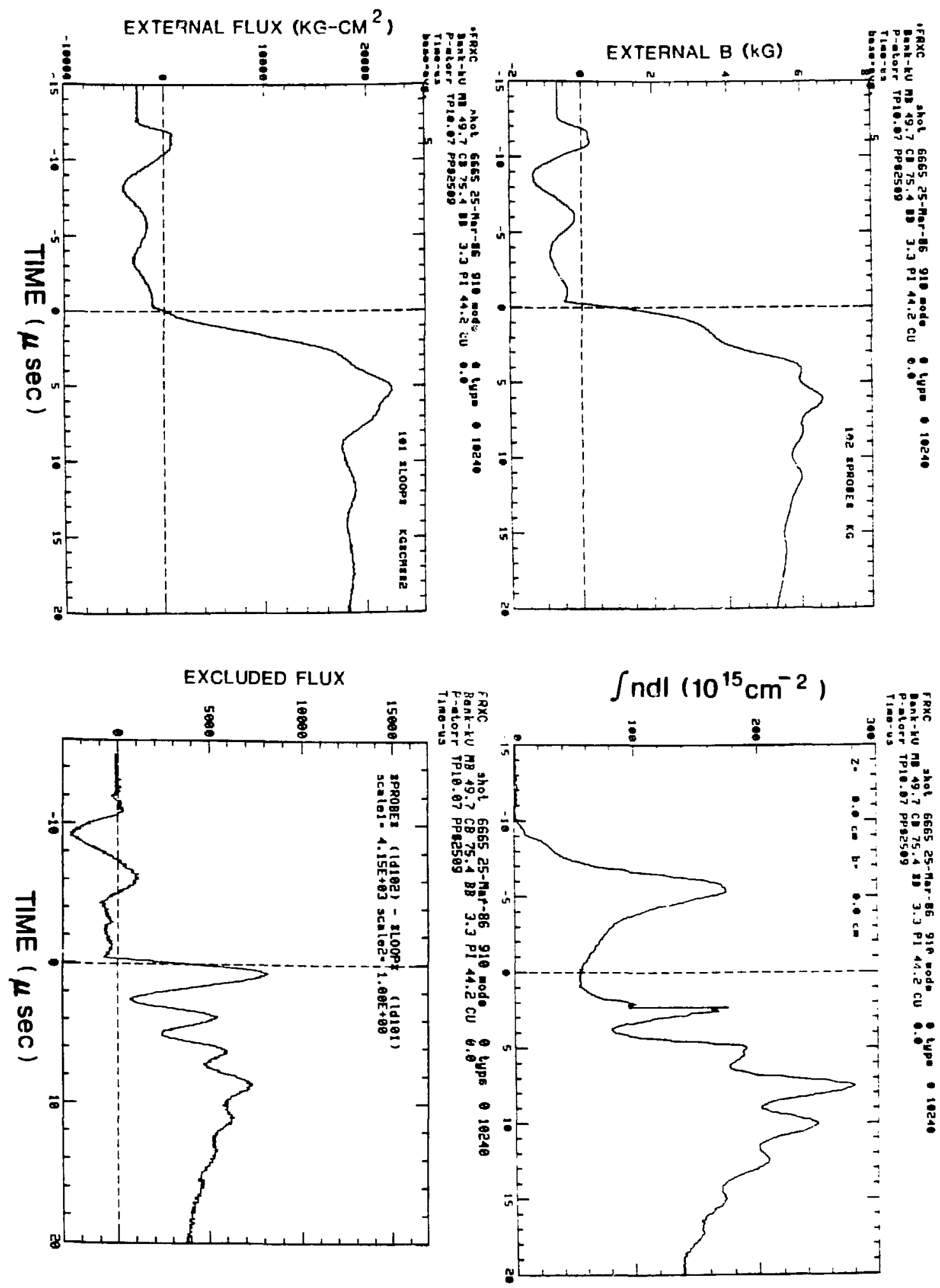

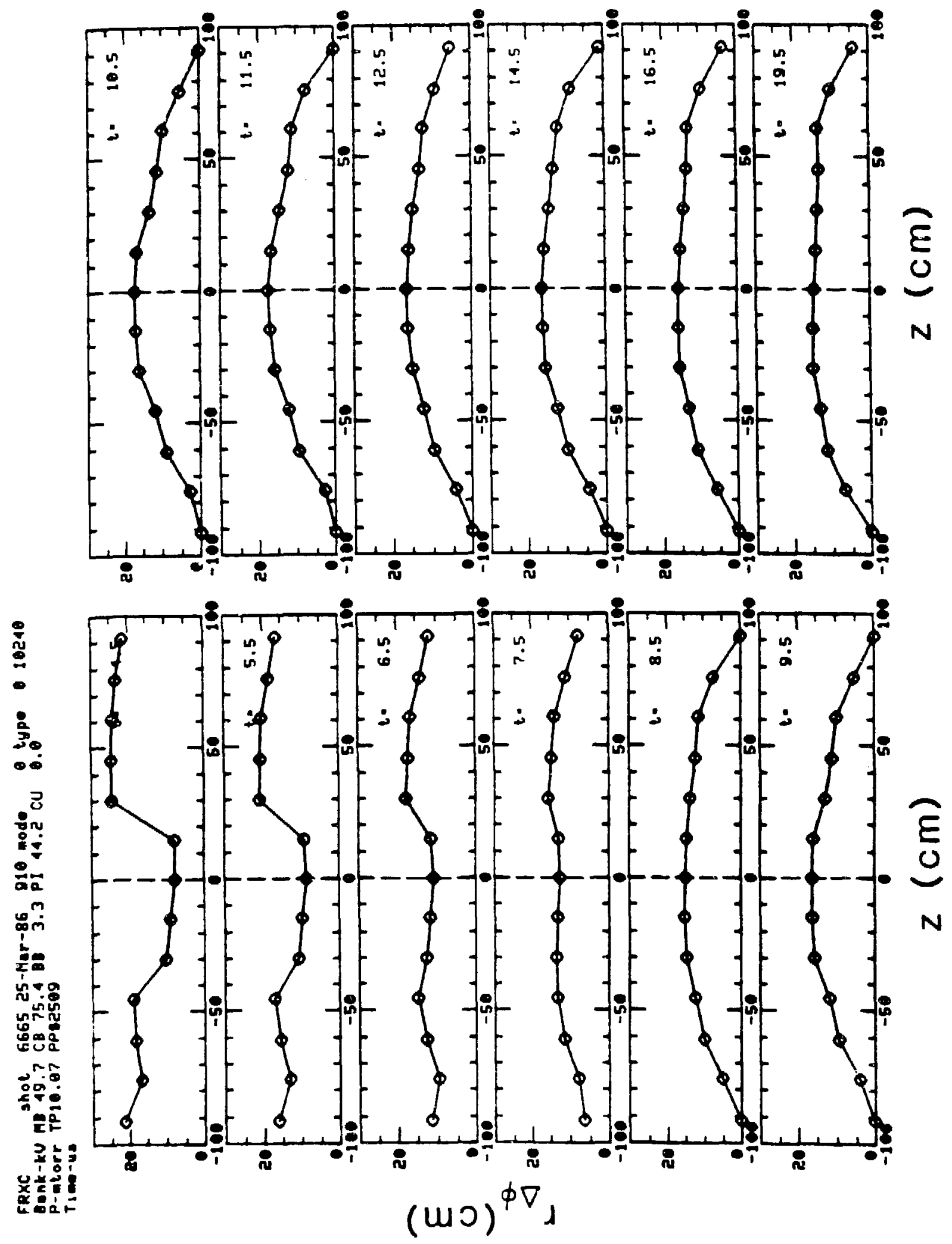

FIG. 11. Time evolution of the excluded flux radius profile during FRC: formation at low bias, $B_{b} \simeq 0.75 \mathrm{kG}$ and $\Delta \tau_{P I}=12 \mu \mathrm{s}$. 


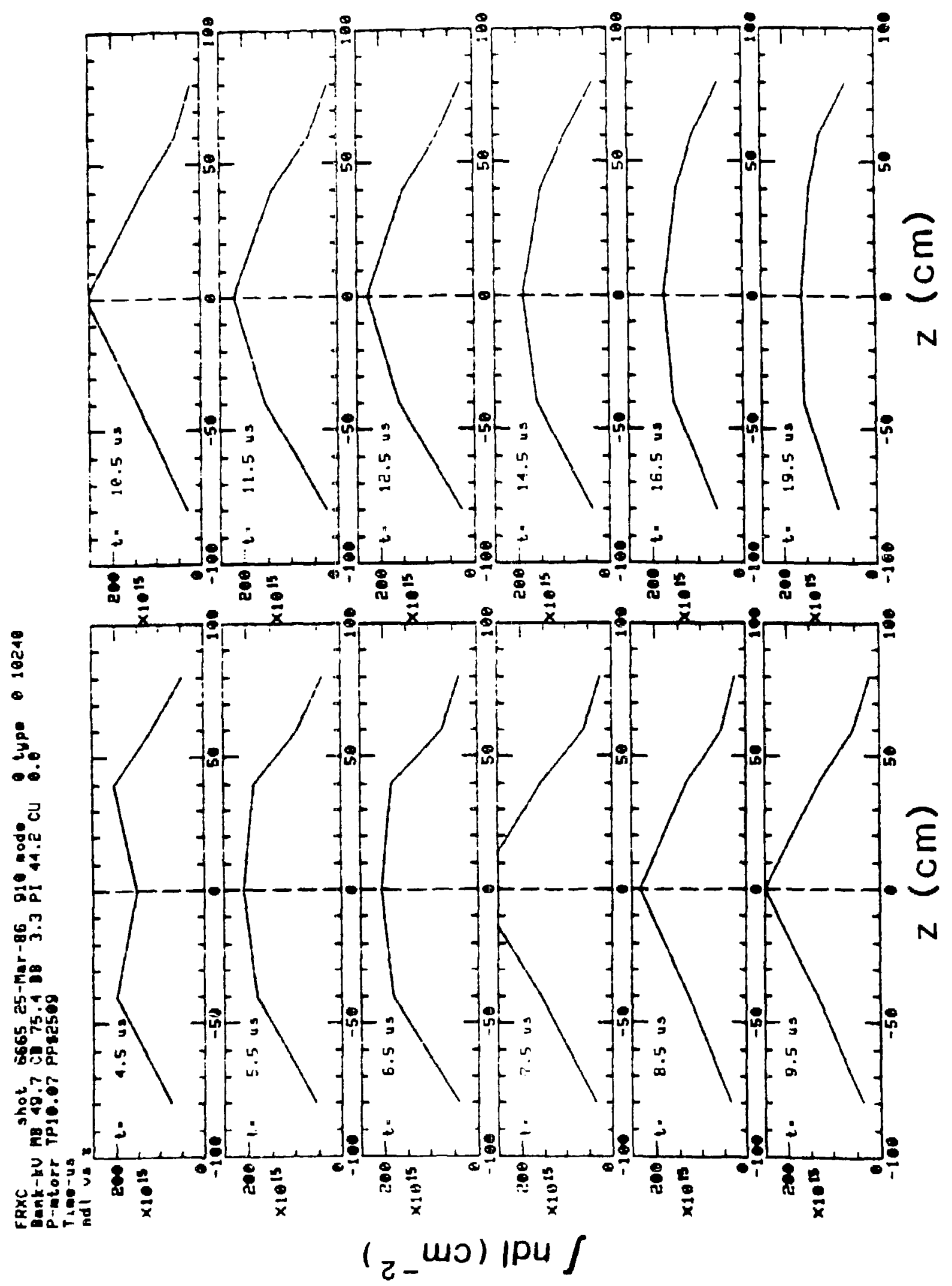

FIG. 12. Time evolution of the axial integral density profile during FRC formation at low bias, $B_{b} \simeq 0.75 \mathrm{kG}$ and $\Delta \tau_{P I}=12 \mu \mathrm{s}$. 


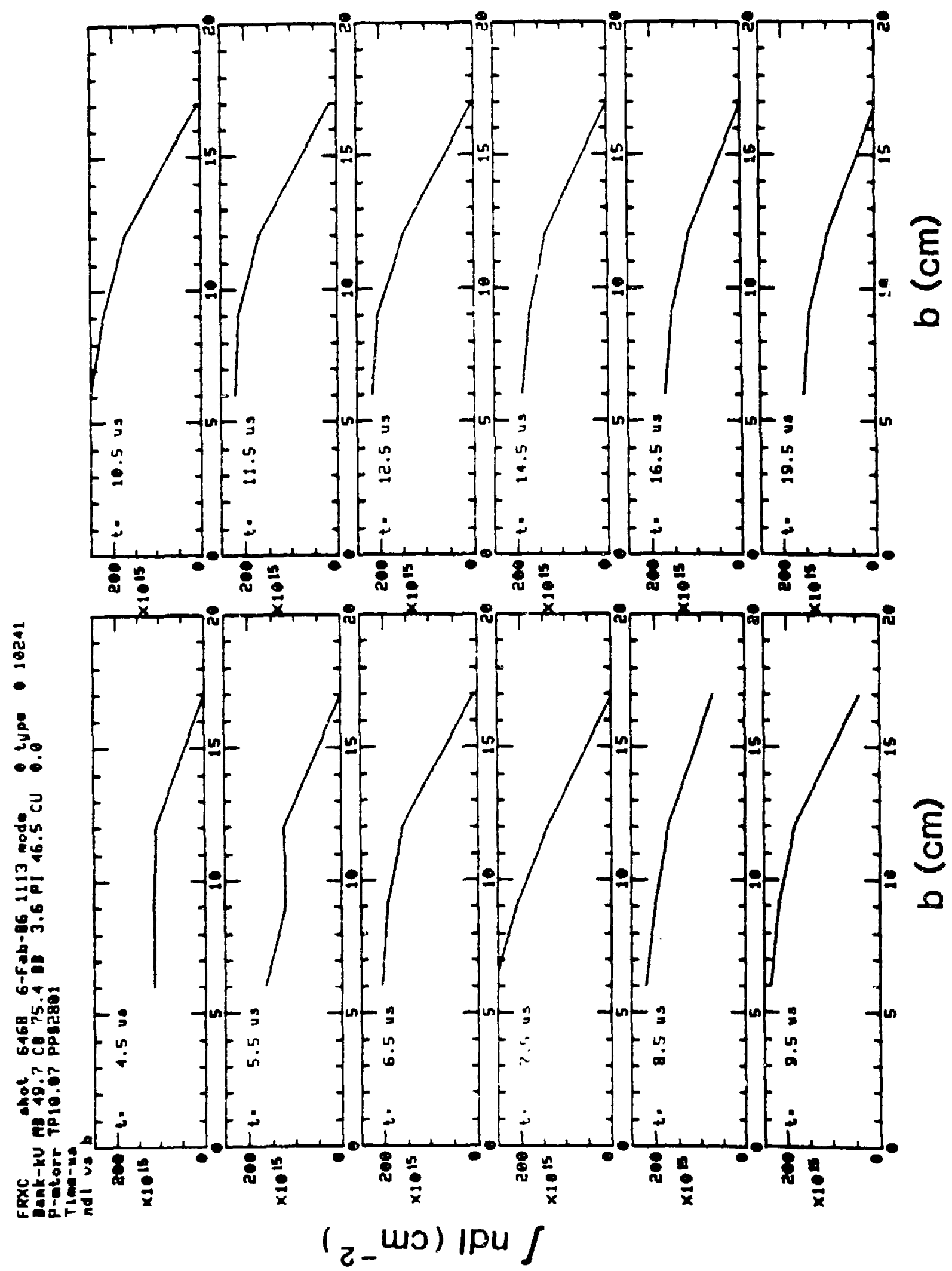

FIG. 13. Time evolution of the radial integral density profile during FRC formation at low bias, $B_{b} \simeq 0.75 \mathrm{kG}$ and $\Delta \tau_{P I}=12 \mu \mathrm{s}$. 


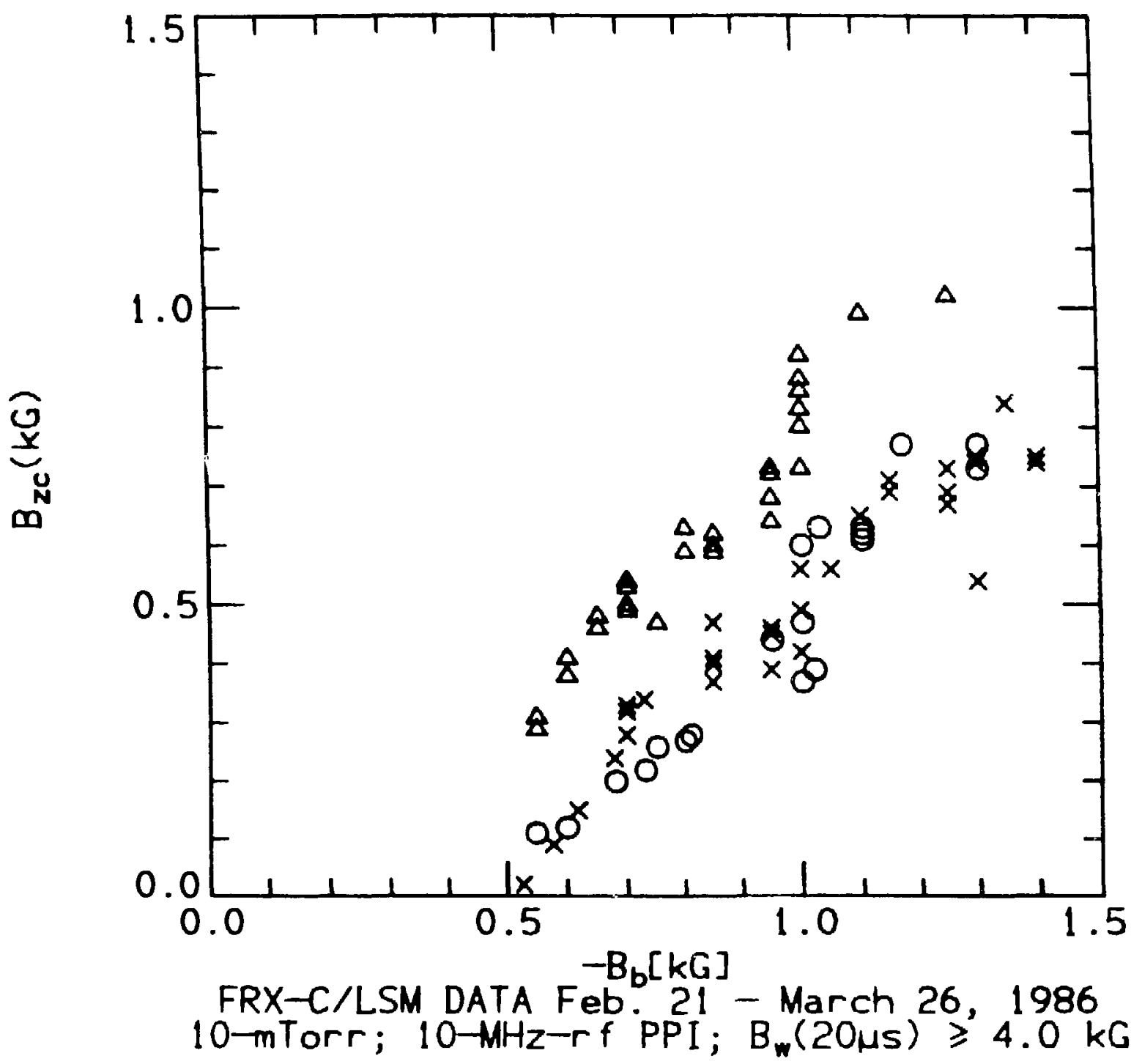

FIG. 14. Variation of "zero-crossing" field, $B_{z c}=\Phi_{t}\left(B_{e x t}=0\right) / \pi r_{t}^{2}$, with bias field for $\Delta \tau_{P I}=5 \mu \mathrm{s}(\Delta), 12 \mu \mathrm{s}(\mathrm{o})$, and $30 \mu \mathrm{s}(x)$. 


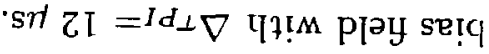

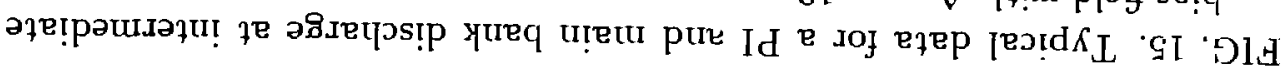
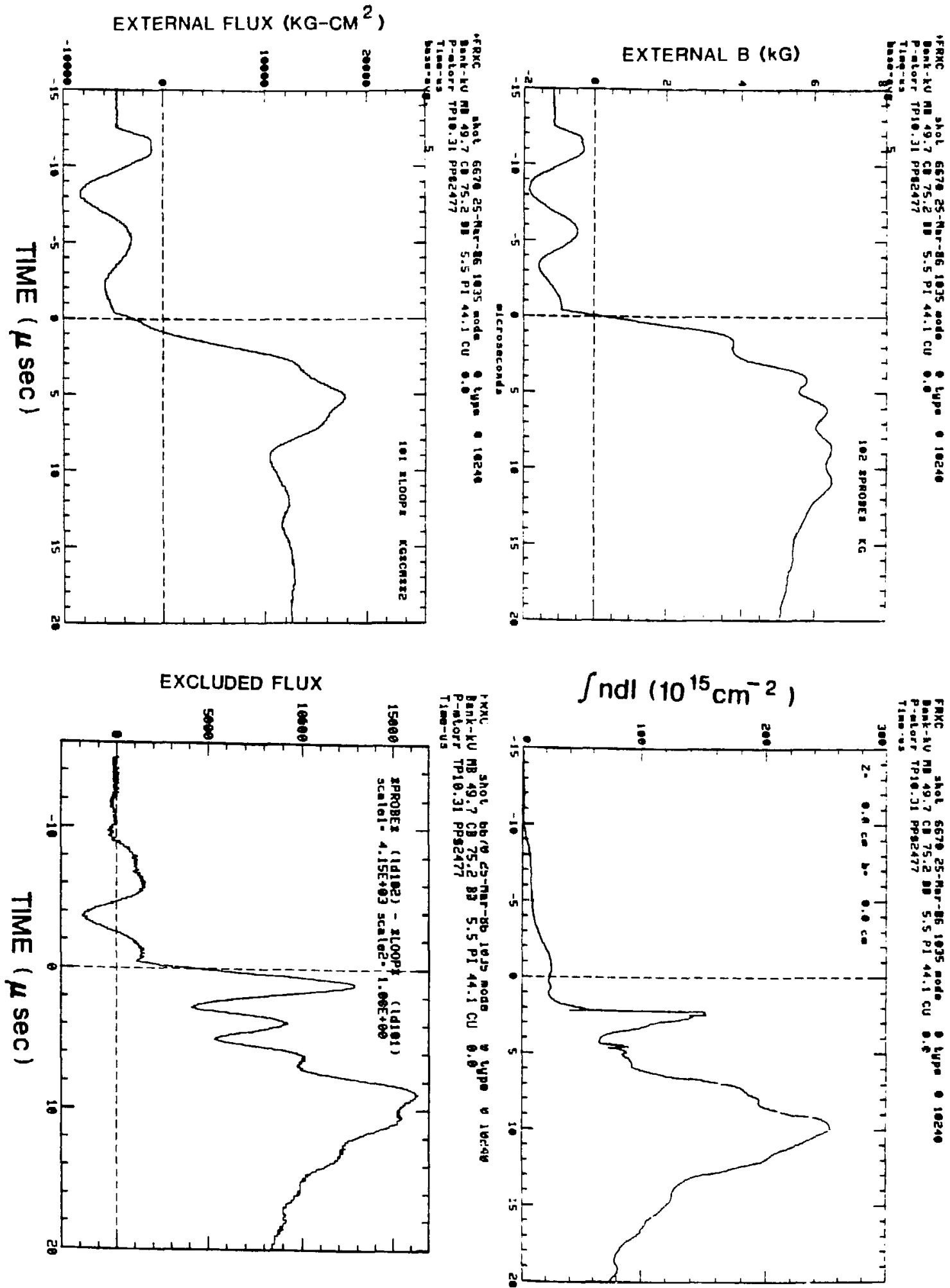

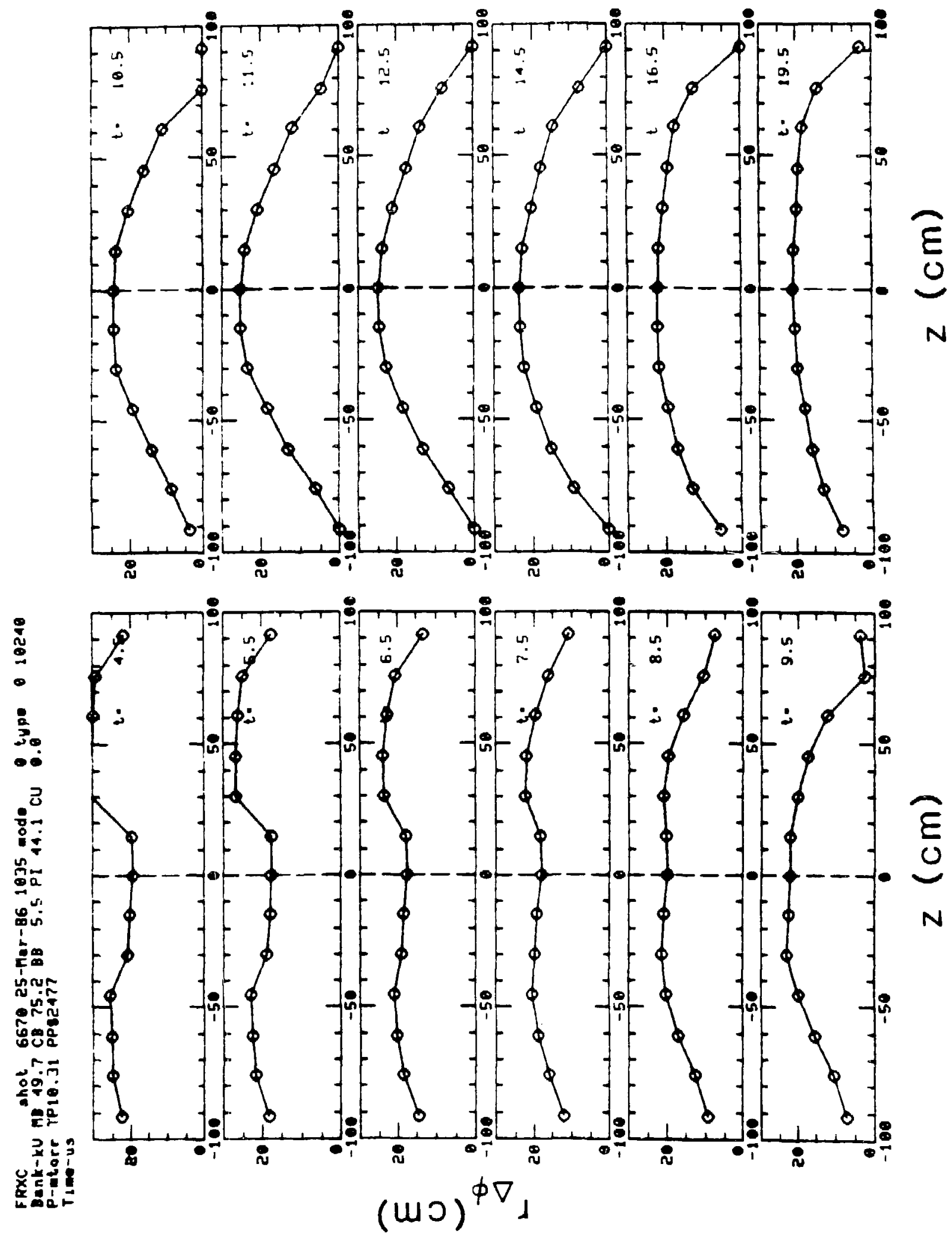

FIC. 16. Time evolution of the excluded fux radius profile during FRC formation at $B_{b}=1.15 \mathrm{kCi}$ and $\Delta \tau_{P I}=12 \mu \mathrm{s}$. 


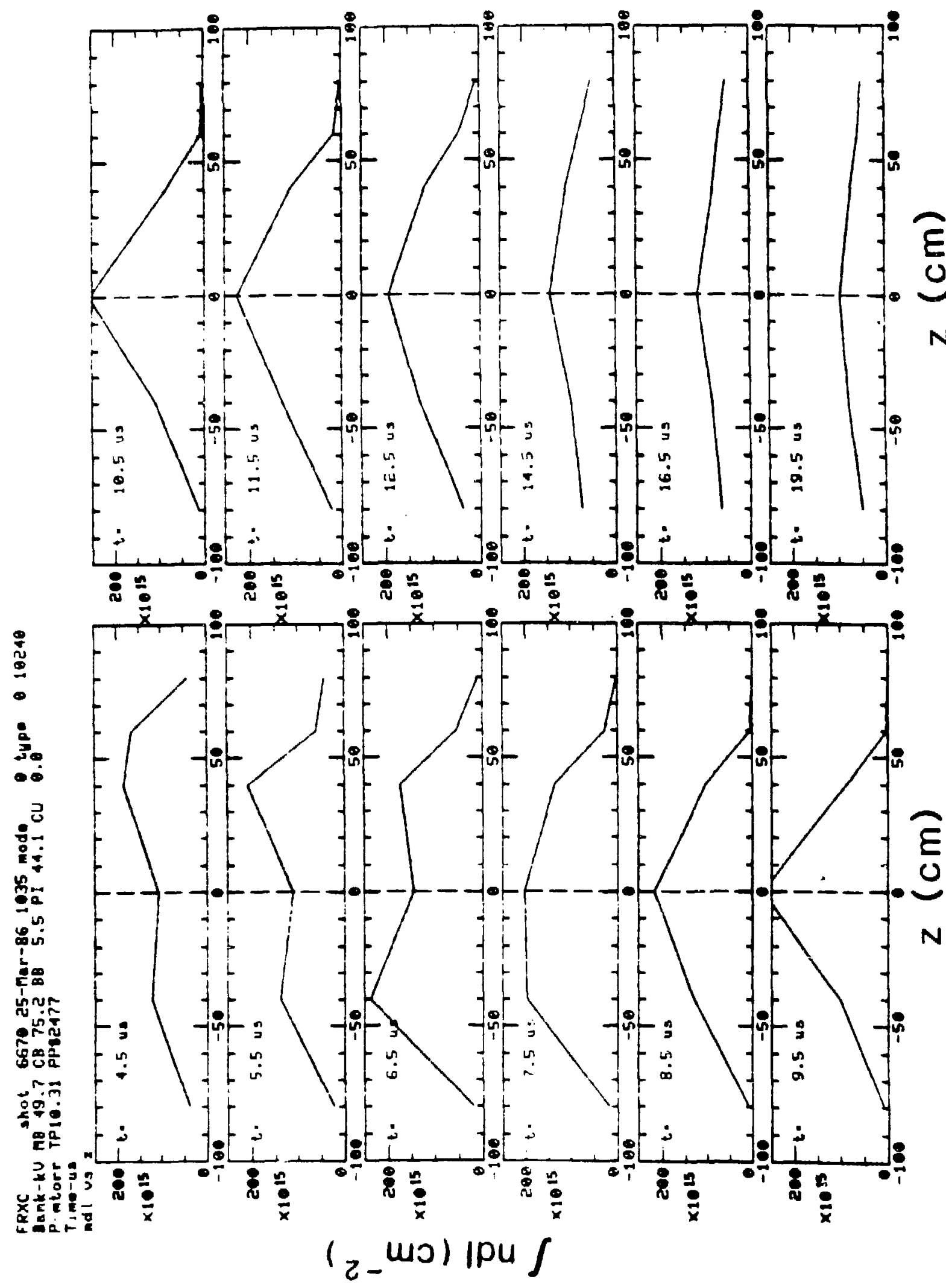

FIG. 17. Time evolution of the axial integral density profile during FRC formation at $B_{b}=1.15 \mathrm{kG}$ and $\Delta \tau_{P I}=12 \mu \mathrm{s}$. 


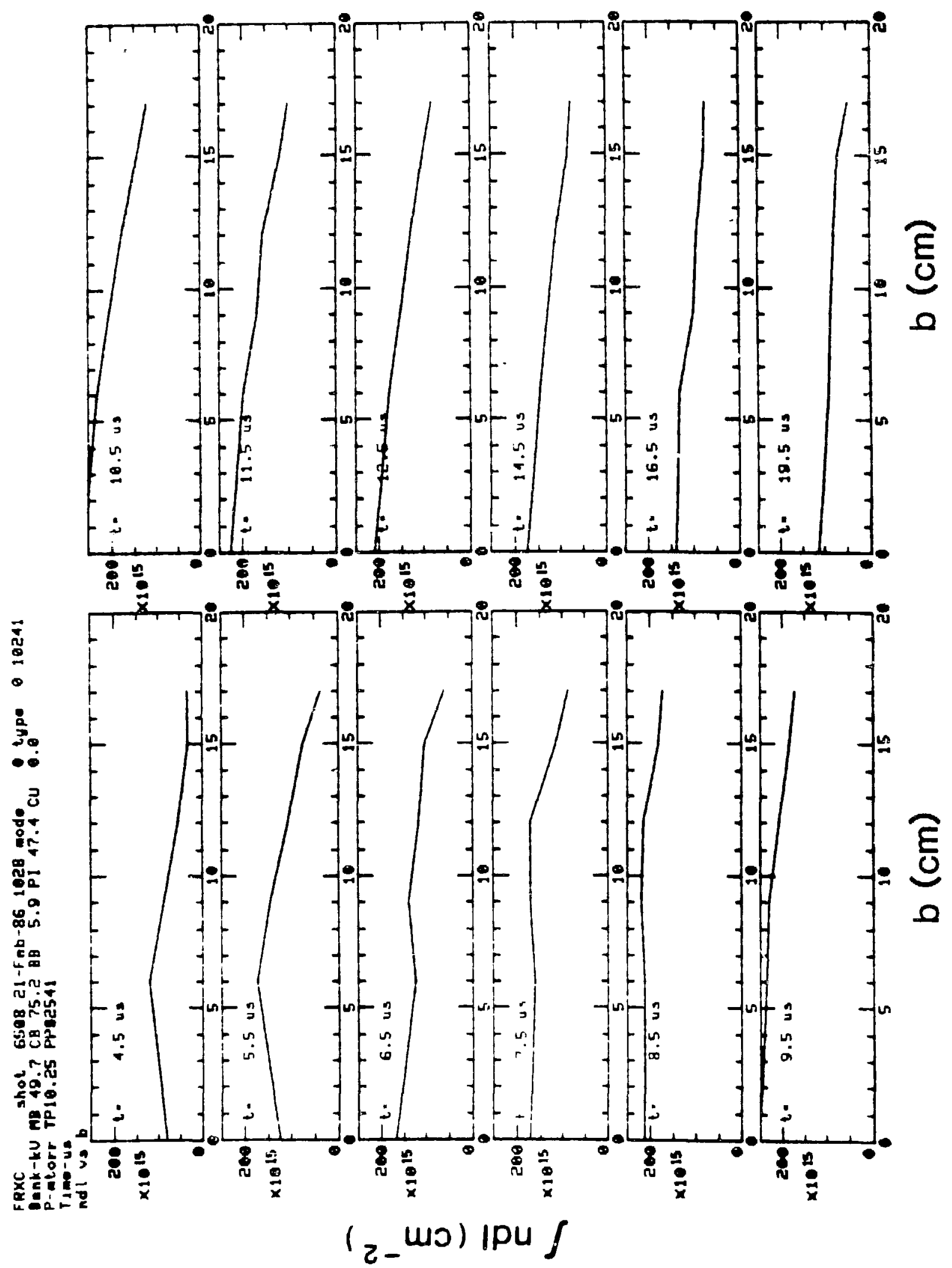

FIG. 18. Time evolution of the radial integral density profile during FRC formation at $B_{b}=1.15 \mathrm{kG}$ and $\Delta \tau_{P I}=12 \mu \mathrm{s}$. 


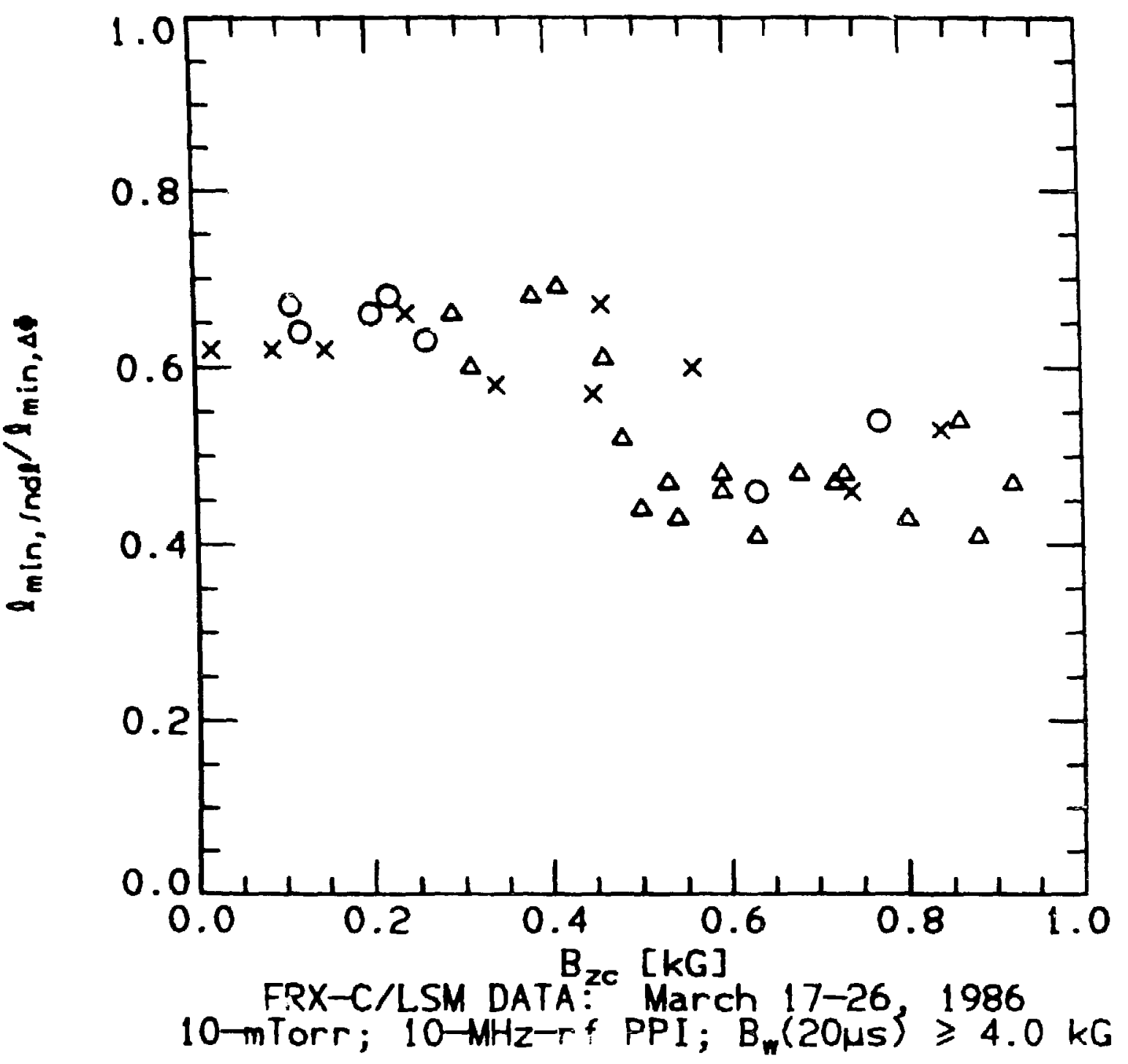

FIG. 19. Ratio of FRC minimum lengths inferred from interferometry and excluded flux plotted against the observed zero-crossing B-field for $\Delta \tau_{P I}=$ $5 \mu \mathrm{s}(\Delta), 12 \mu \mathrm{s}(\mathrm{o})$, and $30 \mu \mathrm{s}(\times)$. 

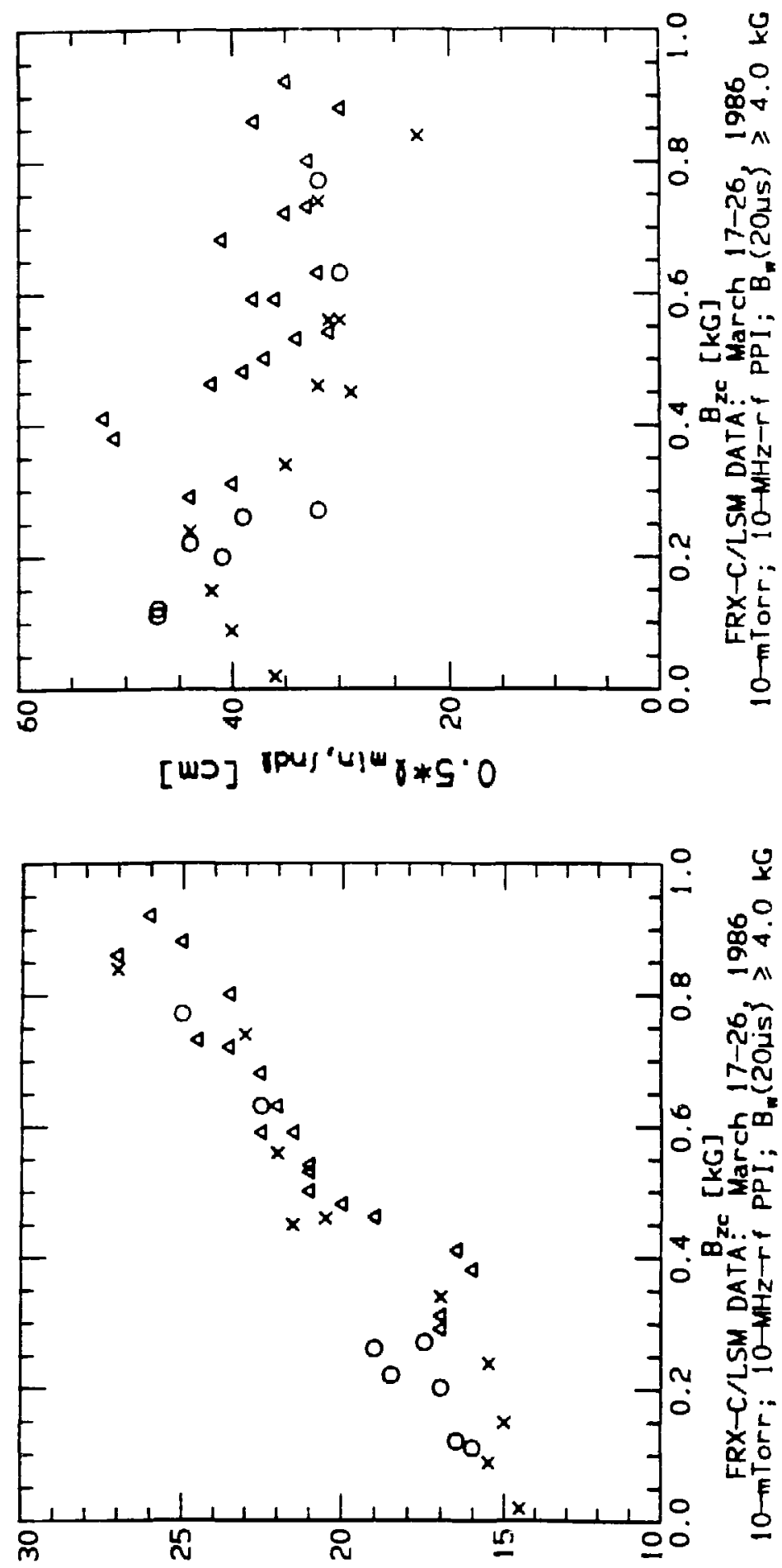

[Wכ]

FIG. 20. The maximum excluded flux radius and minimum integral c'ensity lengths measured during FRC formation plotted against the zerocrossing field for $\Delta \tau_{P I}=5 \mu \mathrm{s}(\Delta), 12 \mu \mathrm{s}(\mathrm{o})$, and $30 \mu \mathrm{s}(\times)$. 


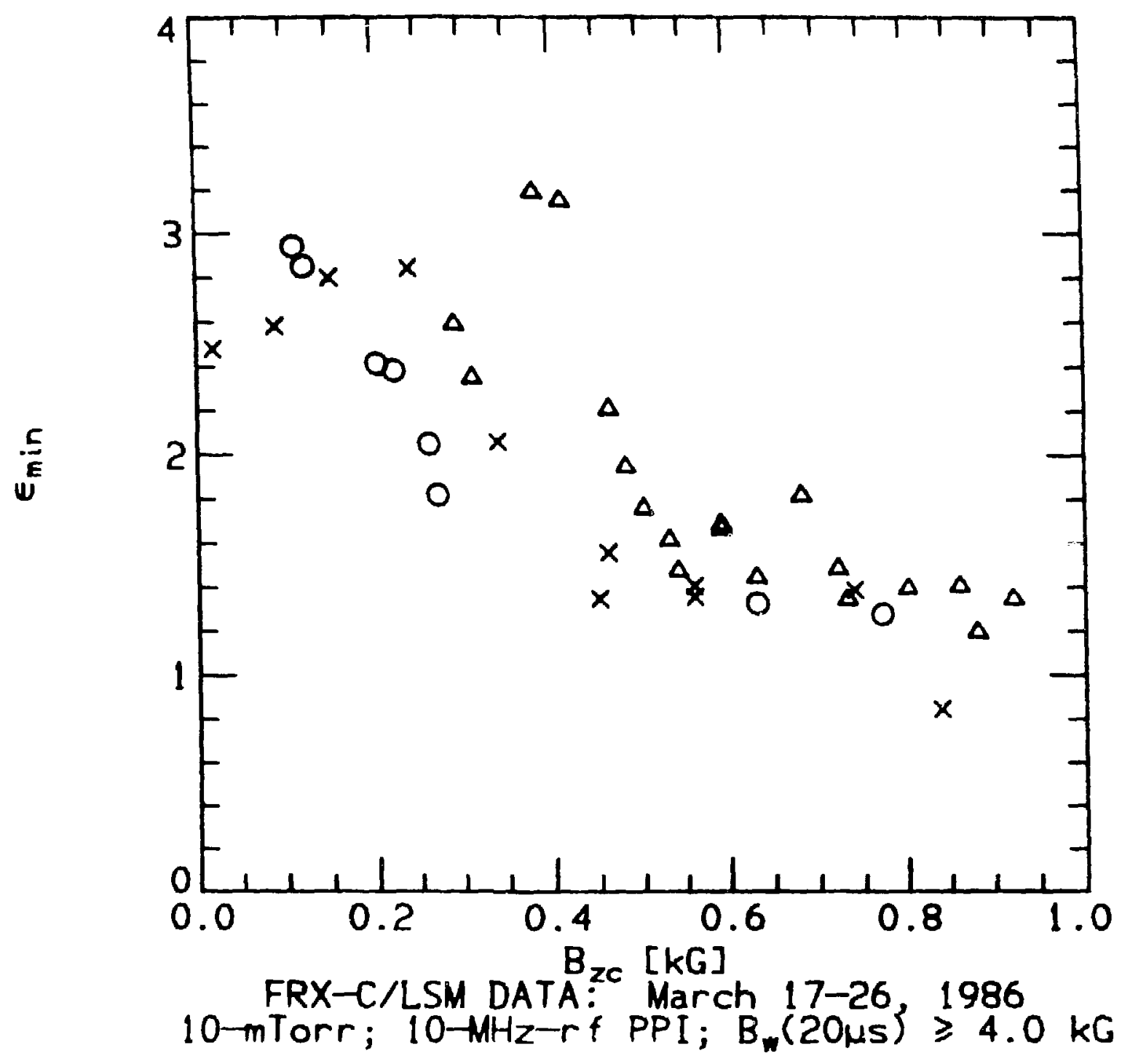

FIG. 21. The variation of the minimum FRC elongation with zero crossing field for $\Delta \tau_{P I}=5 \mu \mathrm{s}(\Delta), 12 \mu \mathrm{s}(\mathrm{o})$, and $30 \mu \mathrm{s}(\times)$. 


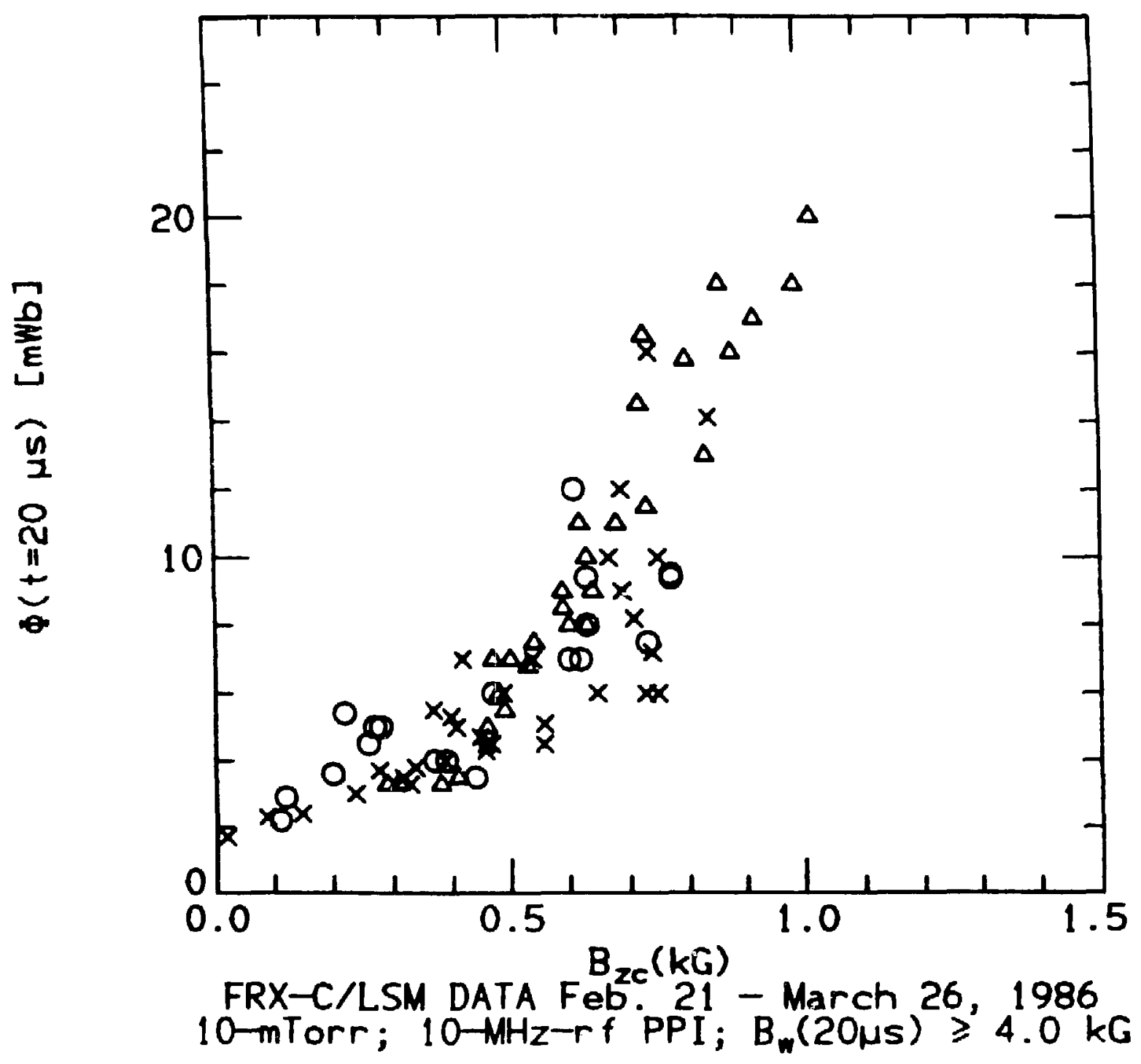

FIG. 22. Variation with $B_{z c}$ of the poloidal flux inferred from measurements at time $\mathrm{t}=20 \mu \mathrm{s}$ using Eq. (1) for $\Delta \tau_{P I}=5 \mu \mathrm{s}(\Delta), 12 \mu \mathrm{s}(\mathrm{o})$, and $30 \mu \mathrm{s}(\times)$. 


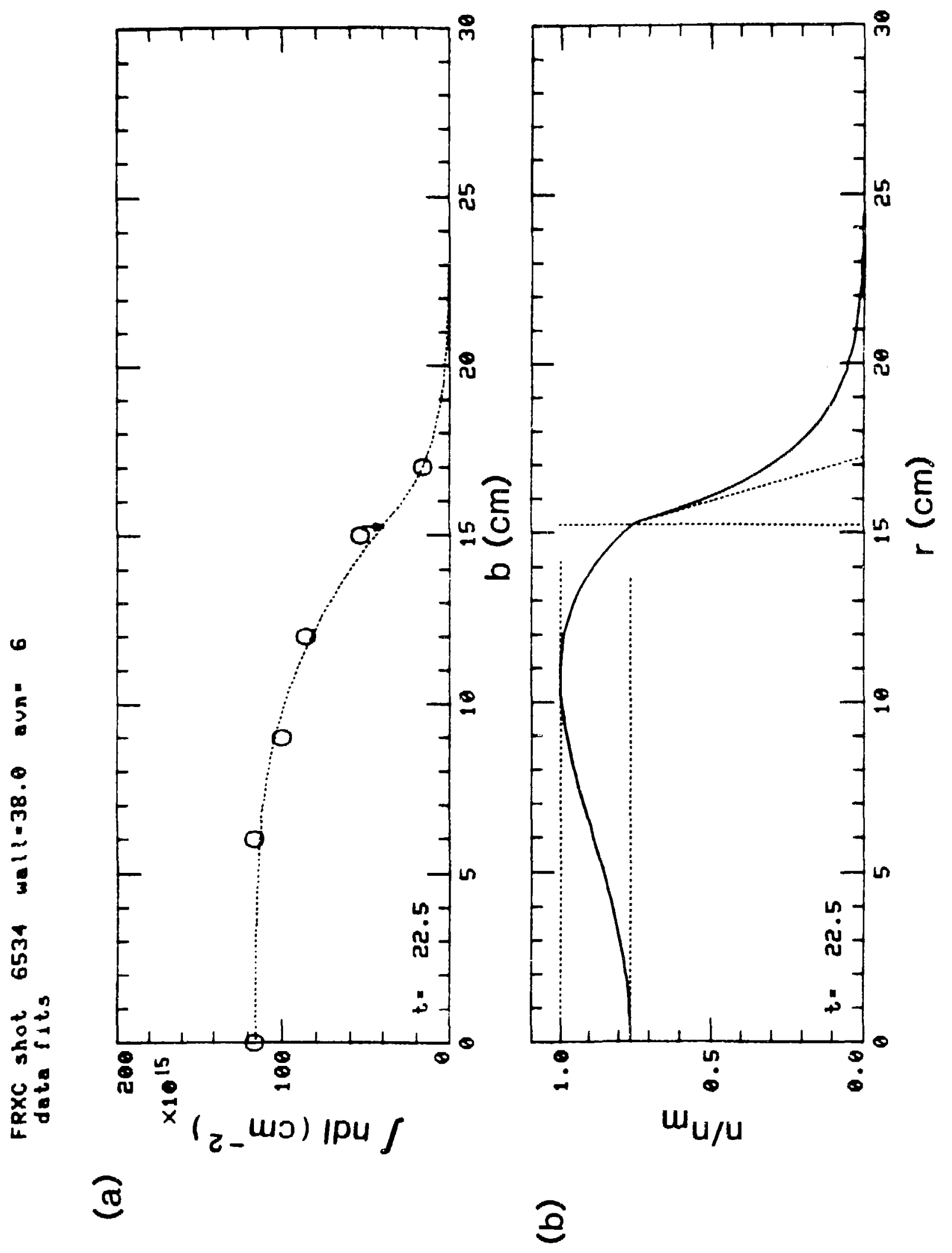

FIG. 23. (a) Radial profile of the integral density measured near the start of the equilibrium phase for a FRC formed at low bias $B_{b}=0.75 \mathrm{kG}$; (b) $n(r)$ profile inferred from Eqs. (2) and (3). 

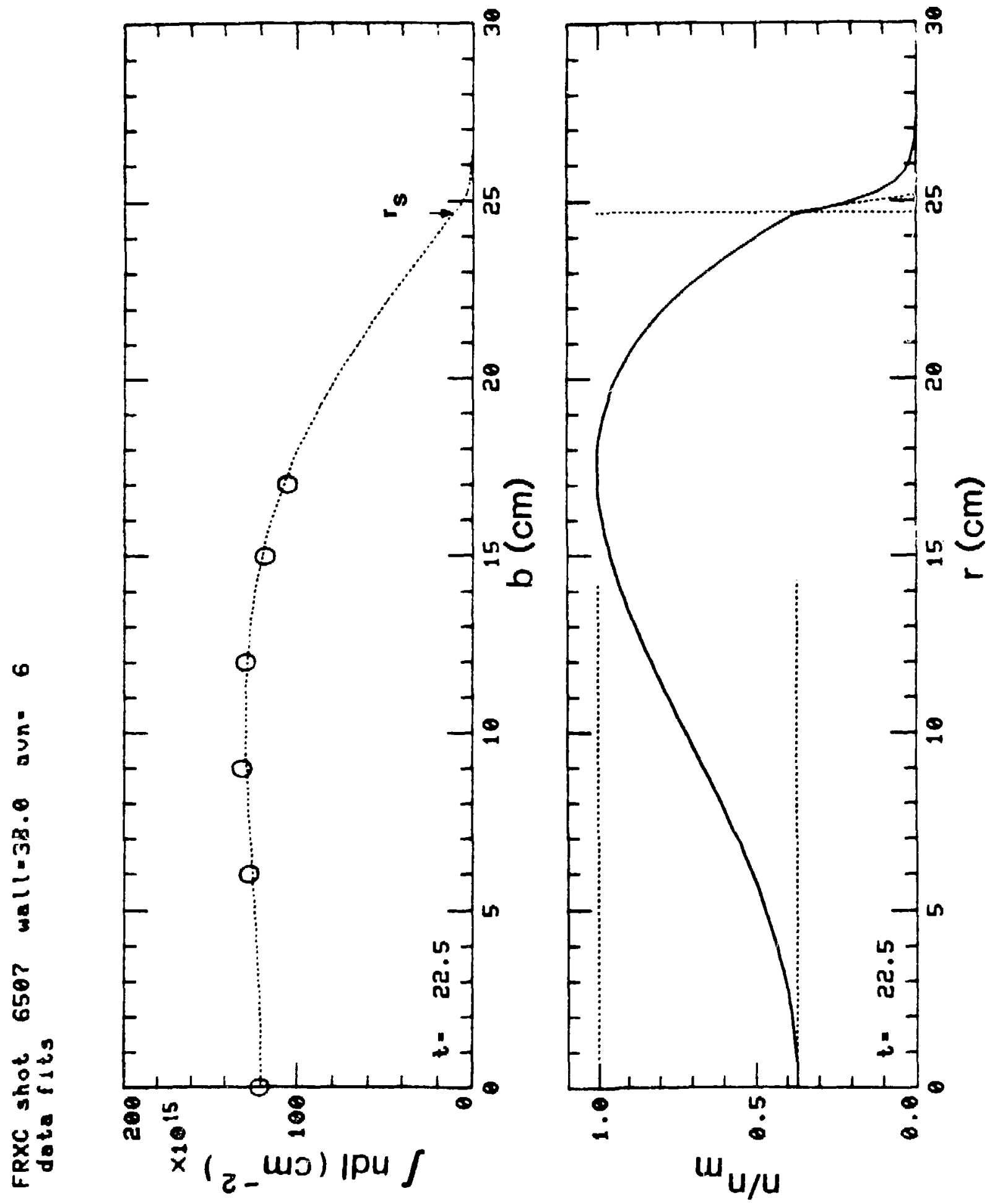

(1)

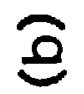

FIG. 24. (a) Radial profile of the integral density measured near the start of the equilibrium phase for a FRC forr ed at high bias, $B_{b} \simeq 1.3 \mathrm{kG}$; (b) $n(r)$ profile inferred from Eqs. (2) and (3). 


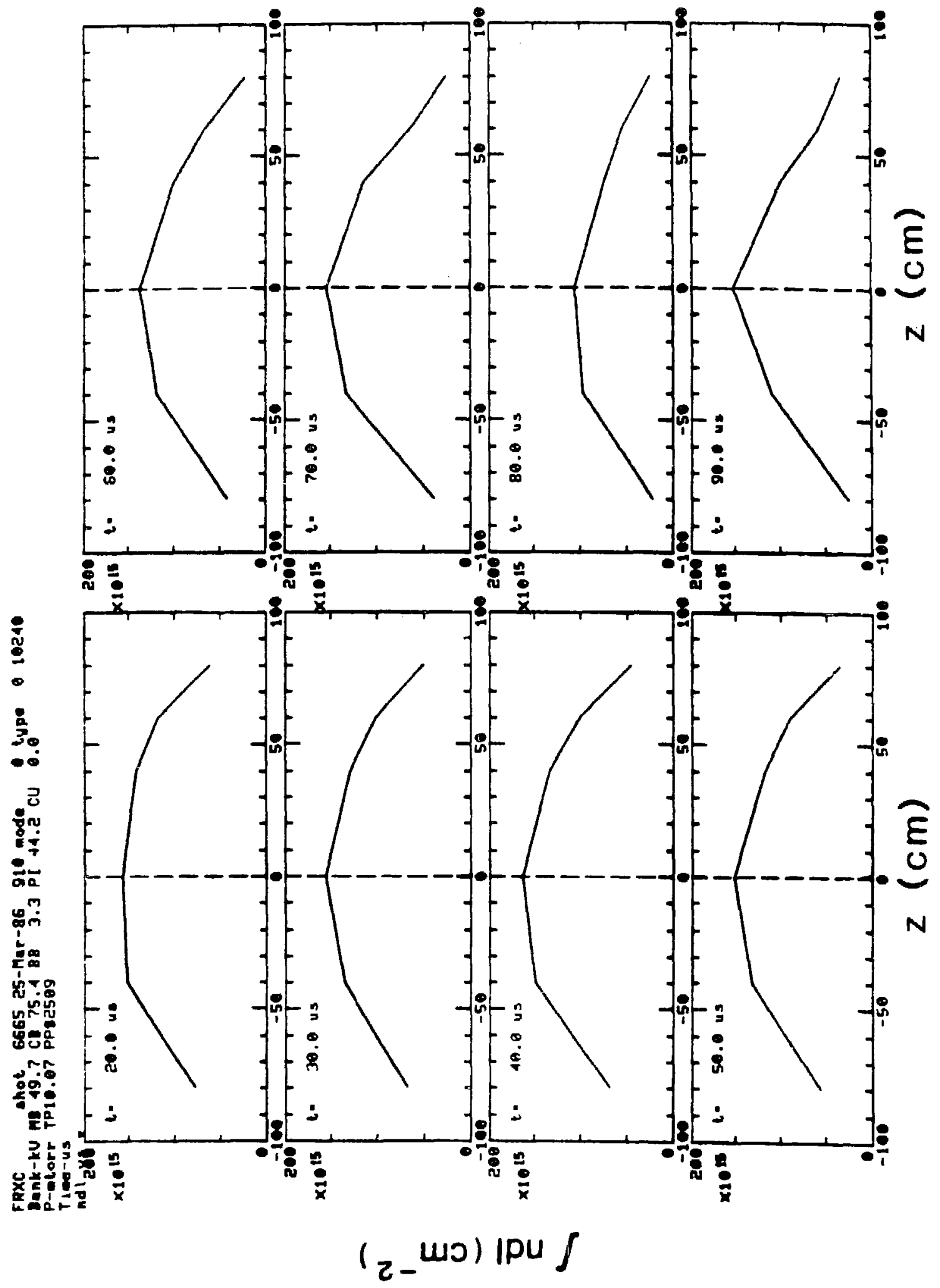

FIG. 25. Time evolution of the axial integral density profile during the equilibrium phase of an FRC formed at low bias $B_{b} \simeq 0.75 \mathrm{kG}$. 

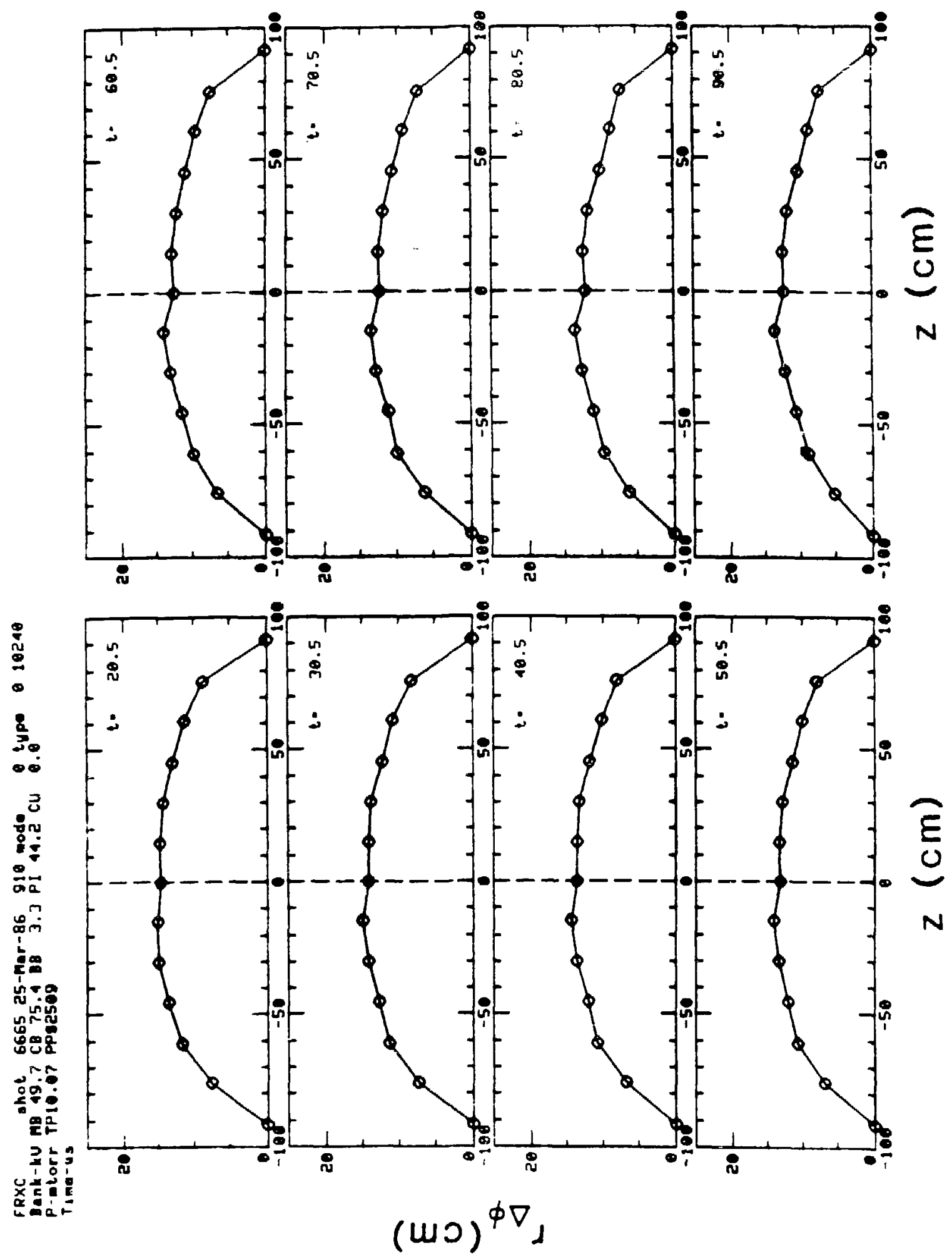

FIG. 26. Time evolution of the excluded flux radius profile during the equilibrium phase of an $\mathrm{FRC}$ formed at low bias, $B_{b} \simeq 0.75 \mathrm{kG}$. 


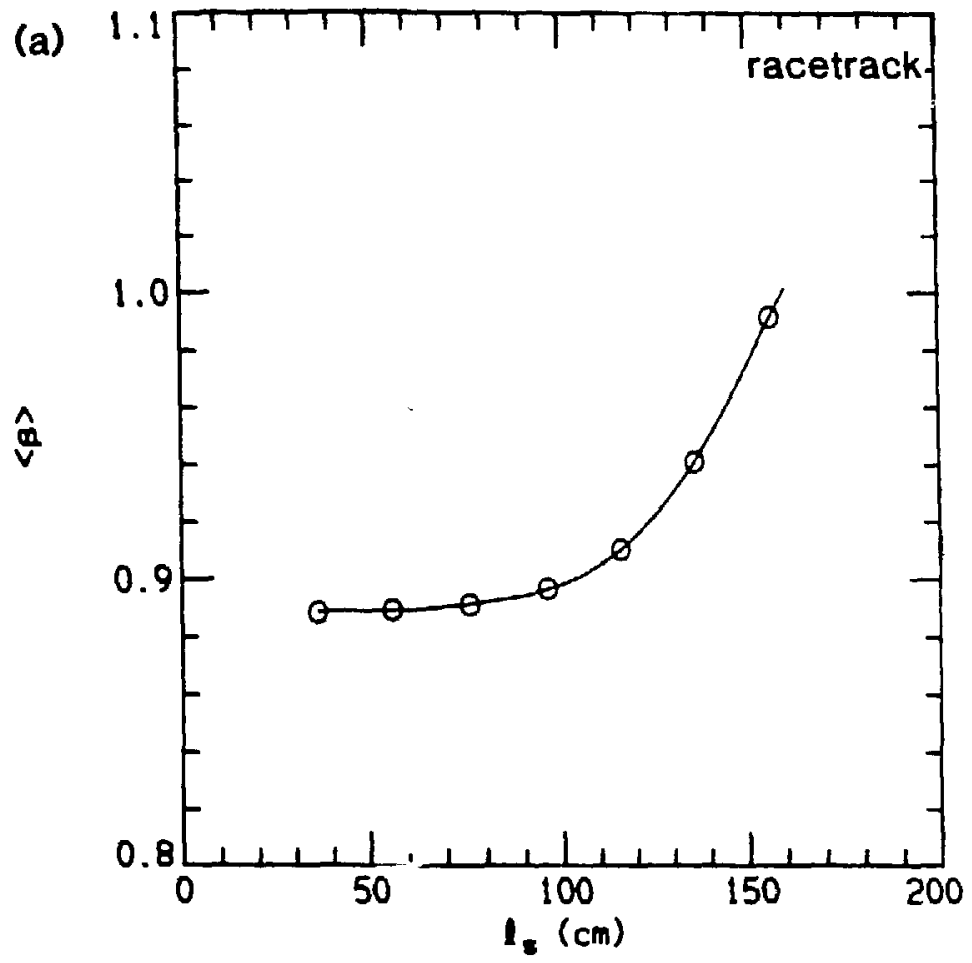

(b)

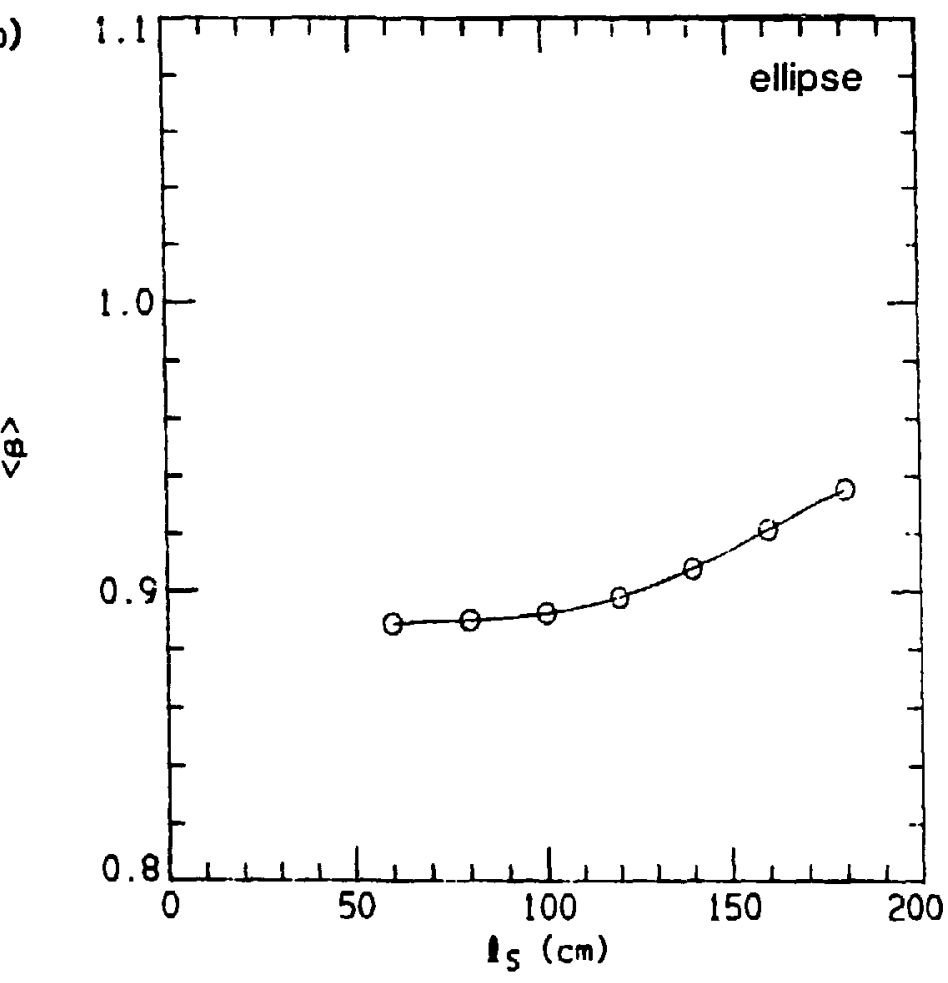

MIRROR EFFECTS ON 〈B> IN LSM: $X_{S}=0.474$

FIG. 27. Changes in $\langle\beta\rangle$ with plasma length computed for (a) "racetrack" and (b) elliptical FRC separatrices. The length of the straight central section of the LSM $\theta$-pinch coil is $140 \mathrm{~cm}$. 

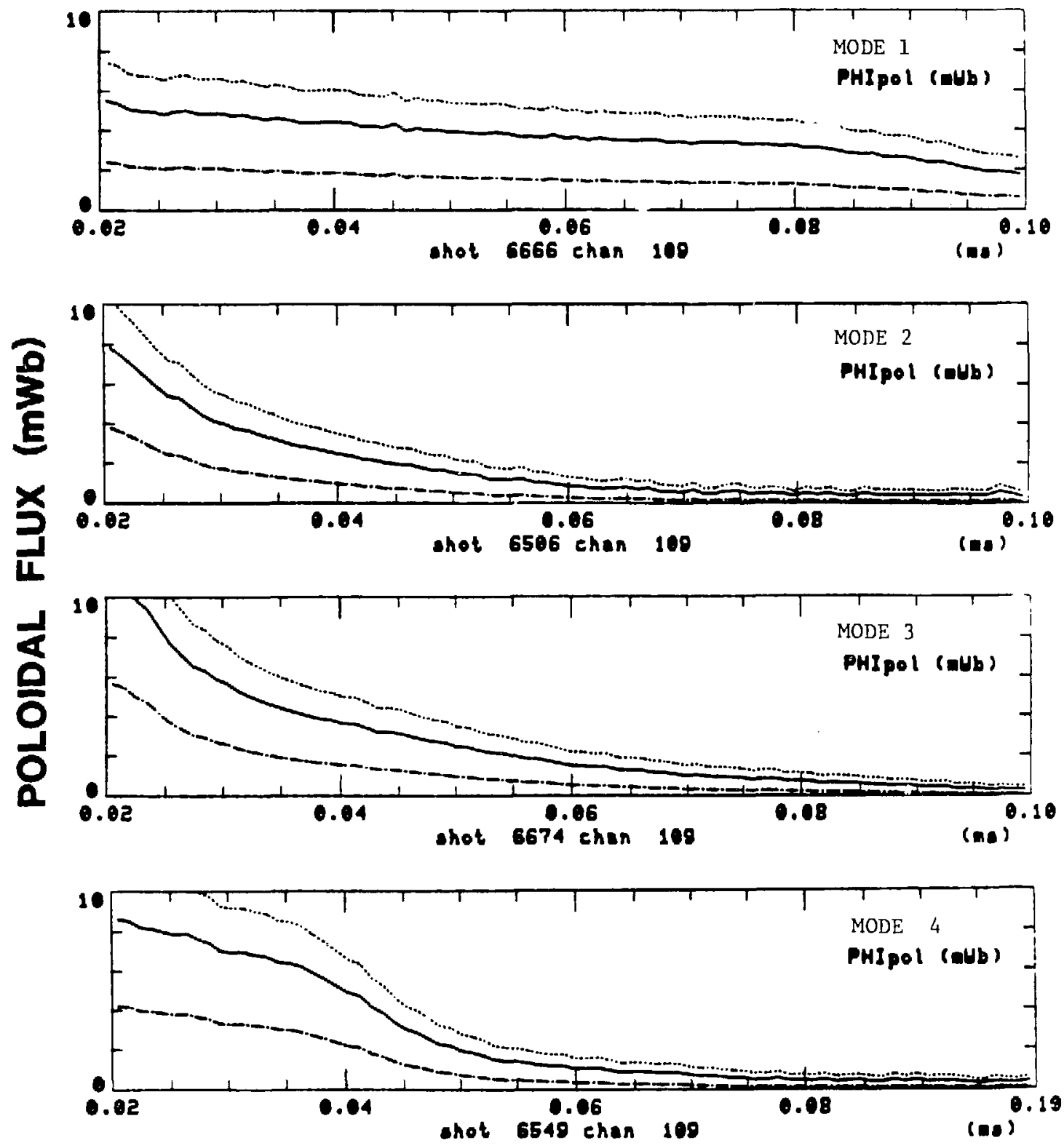

TIME (msec)

FIG. 28. Typical $\Phi_{p}(\mathrm{t})$ data for four discharges inferred from external magnetic measurements and Eqs. (1), (4), and (5). 

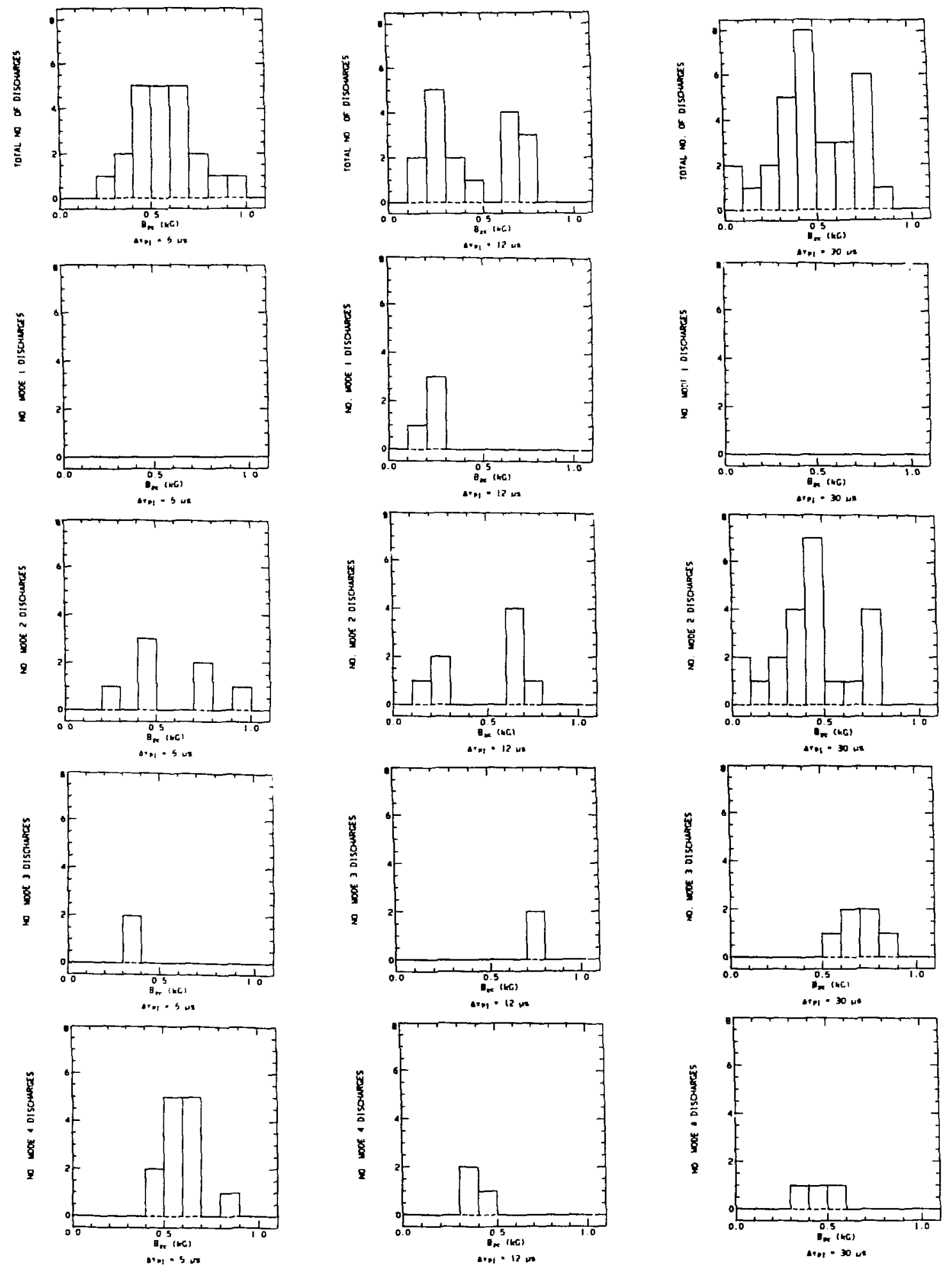

FIG. 29. Histograms illustrating the relative frequency of occurrence for the four flux decay modes. 


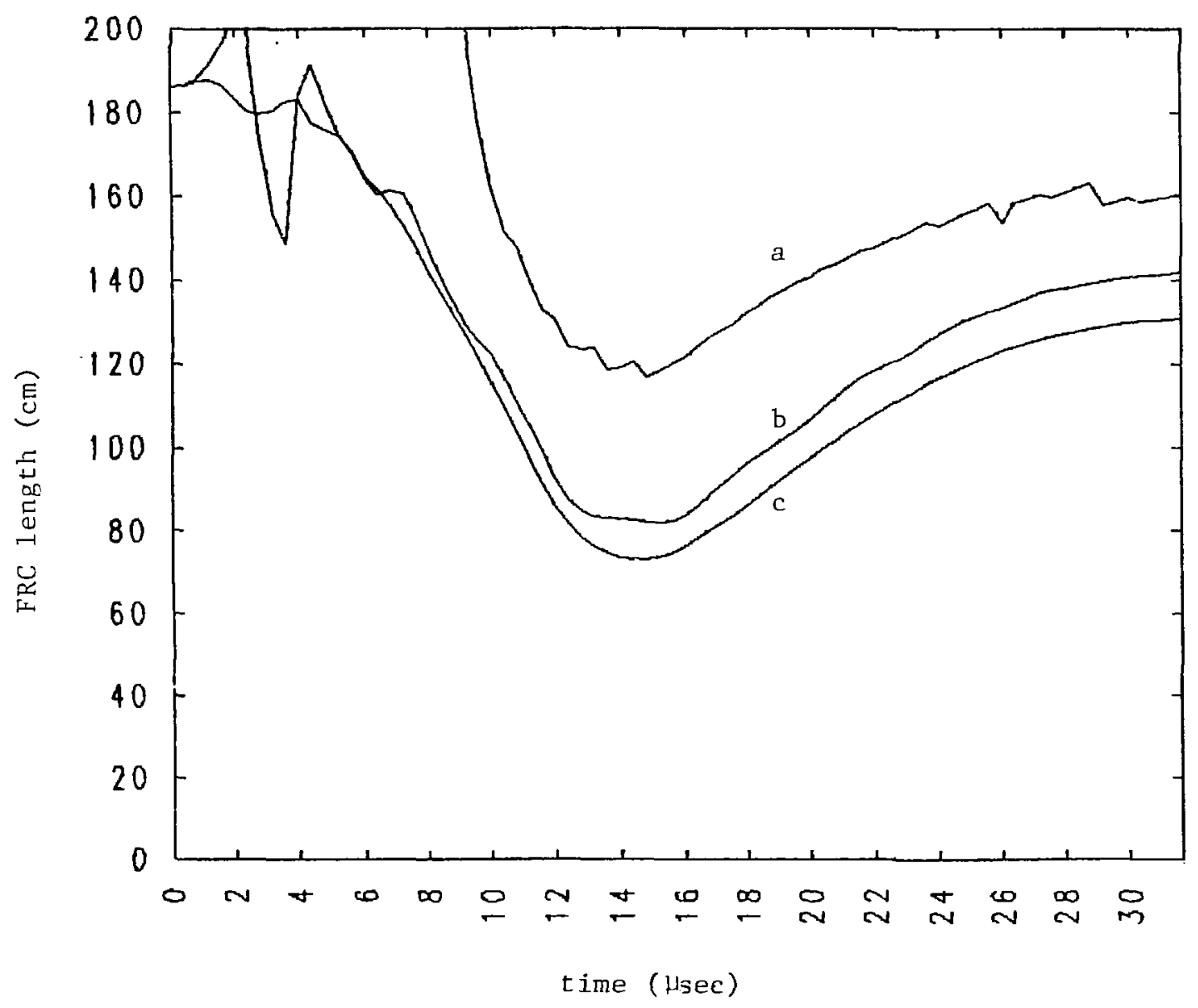

FIG. 30. Variation of the FRC length as estimated using: a) the excluded flux radius, b) $\int \mathrm{ndl}$, and c) the line density for a typical MHD simulation of FRC formation in LSM. 


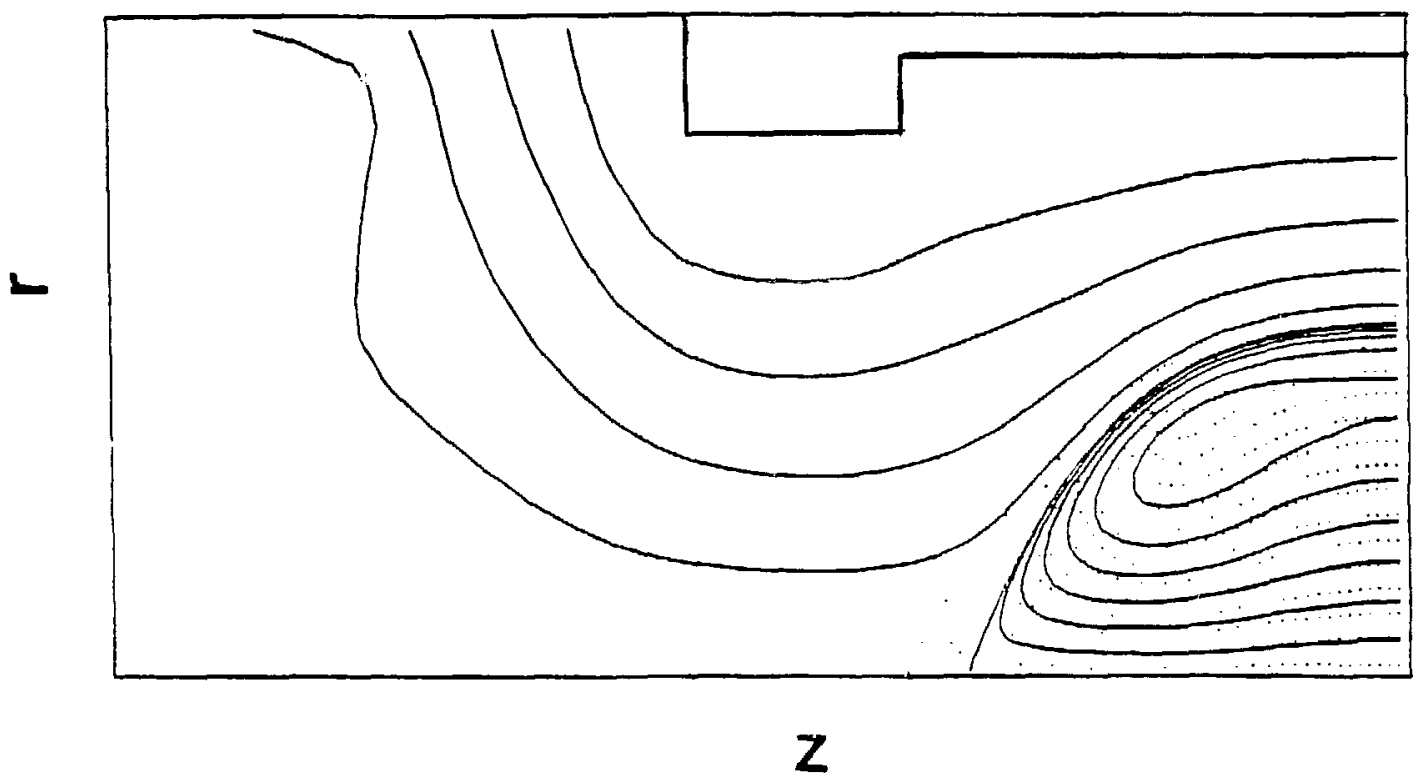

\section{(b) DENSITY CONTOURS}

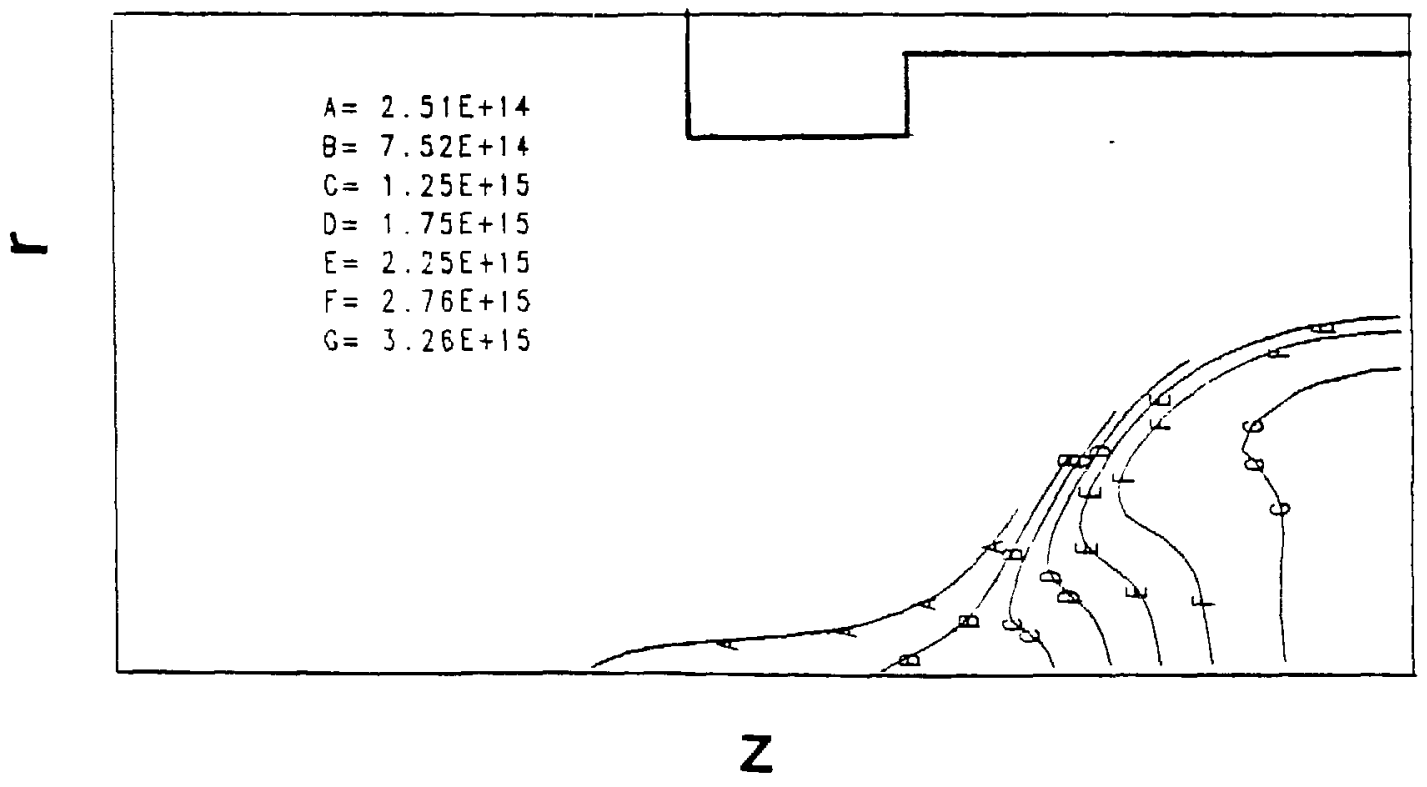

FIG. 31. The magnetic flux contours and the electron density contour at $t$ $=16 \mu \mathrm{s}$ for the same MHD simulation as in Fig. 30 . 